\title{
An X-ray/SDSS sample
}

\section{Multi-phase outflow incidence and dependence on AGN luminosity}

\author{
M. Perna ${ }^{1,2,3}$, G. Lanzuisi ${ }^{1,2}$, M. Brusa ${ }^{1,2}$, M. Mignoli ${ }^{2}$, and G. Cresci $^{3}$ \\ 1 Dipartimento di Fisica e Astronomia, Università di Bologna, viale Berti Pichat 6/2, 40127 Bologna, Italy \\ e-mail: michele.perna4@unibo.it \\ 2 INAF-Osservatorio Astronomico di Bologna, via Ranzani 1, 40127 Bologna, Italy \\ 3 INAF-Osservatorio Astrofisico di Arcetri, Largo Enrico Fermi 5, 50125 Firenze, Italy
}

Received 29 December 2016 / Accepted 13 March 2017

\begin{abstract}
Aims. The connection between the growth of super-massive black holes (SMBHs) and the evolution of their host galaxies is nowadays well established, although the underlying mechanisms explaining their mutual relations are still debated. Multi-phase fast, massive outflows have been postulated to play a crucial role in this process. The aim of this work is to constrain the nature and the fraction of outflowing gas in active galactic nuclei (AGNs) as well as the nuclear conditions possibly at the origin of such phenomena.

Methods. We present a large spectroscopic sample of X-ray detected SDSS AGNs at $z<0.8$ with a high signal-to-noise ratio in the [O III] $\lambda 5007$ line to unveil the faint wings of the emission profile associated with AGN-driven outflows. We used X-ray and optical flux ratio diagnostics to select the sample. We derived physical and kinematic characterization by re-analysing optical (and X-ray) spectra.

Results. We derive the incidence of ionized $(\sim 40 \%)$ and atomic $(<1 \%)$ outflows covering a wide range of AGN bolometric luminosity from $10^{42}$ to $10^{46} \mathrm{erg} / \mathrm{s}$. We also derive bolometric luminosities and X-ray bolometric corrections to test whether the presence of outflows is associated with an X-ray loudness, as suggested by our recent results obtained by studying high- $z$ QSOs.

Conclusions. We study the relations between the outflow velocity inferred from [O III] kinematic analysis and different AGN power tracers, such as black hole mass $\left(M_{\mathrm{BH}}\right),[\mathrm{O} \mathrm{III}]$, and X-ray luminosity. We show a well-defined positive trend between outflow velocity and $L_{\mathrm{X}}$, for the first time, over a range of 5 order of magnitudes. Overall, we find that in the QSO-luminosity regime and at $M_{\mathrm{BH}}>10^{8} M_{\odot}$ the fraction of AGNs with outflows becomes $>50 \%$. Finally, we discuss our results about X-ray bolometric corrections and outflow incidence in cold and ionized phases in the context of an evolutionary sequence allowing two distinct stages for the feedback phase: first, an initial stage characterized by X-ray/optical obscured AGNs, in which the atomic gas is still present in the ISM and the outflow processes involve all the gas components and, second, a later stage associated with unobscured AGNs, in which the line of sight has been cleaned and the cold components have been heated or exhausted.
\end{abstract}

Key words. galaxies: active - quasars: emission lines - ISM: jets and outflows

\section{Introduction}

Feedback mechanisms during the bright phase of active galactic nuclei (AGN) are indicated as the leading processes responsible for the joint evolution of super-massive black holes (SMBHs) and galaxies (e.g. Fabian 2012; King \& Pounds 2015).

Evolutionary models for the co-eval growth of galaxies and AGN predict a relatively short $(\ll 500 \mathrm{Myr})$ three stage phase triggered by major merger events for the quasar population $\left(L_{\text {bol }}>10^{45} \mathrm{erg} / \mathrm{s}\right.$; Menci et al. 2008; Hopkins et al. 2008). In this evolutionary framework, the funnelling of a large amount of gas into the nuclear region ignites a concomitant growth of the central SMBH and the host bulge through efficient star formation (SF) in a dust-enshrouded environment of dense gas. When the SMBH achieves a critical mass, establishing the $M-\sigma$ relation, it becomes powerful enough to affect the host galaxy. In the case of high accretion rates, the energy output of the SMBH couples to the different phases of the interstellar medium (ISM). During this coupling, galaxy-wide winds are expected to quench the galaxy SF by reheating the gas or pushing it out of the galactic potential well.
The presence of AGN-driven outflows is now very well established through high resolution observations of local and high-redshift galaxies at different wavelengths tracing the different phases (ionized, neutral, and molecular) of the gas in the ISM (e.g. Brusa et al. 2015; Cimatti et al. 2013; Feruglio et al. 2010; Glikman et al. 2012; Lanzuisi et al. 2015; Liu et al. 2013; Rupke et al. 2005b; Sturm et al. 2011; Talia et al. 2016; Villar-Martín et al. 2014). Moreover, with the advent of high resolution, sensitive integral field spectrographs and millimetre interferometers, it is now possible to study in detail the feedback phenomena characterizing the galaxy-wide extension and morphology of the ejected material as well as the masses and the energetics related to outflows (e.g. Brusa et al. 2016; Carniani et al. 2015; Cicone et al. 2014; Cresci et al. 2015a; Harrison et al. 2012, 2014; Perna et al. 2015a,b). However, both the triggering feedback mechanisms and physical processes responsible for the coupling between the AGN winds and ISM, resulting in outflows, remain largely unknown. The full characterization of the AGN-host galaxy system is needed to discriminate between the details of various model realizations (see e.g. the detailed discussion in Brusa et al. 2015). Multiwavelength data are essential to derive nuclear and host properties associated with 
the presence of outflows, while synergies between major facilities (e.g. ALMA, NOEMA, SINFONI, and MUSE) are required to study the multiphase wind/ISM interactions. In this respect, we note that spatially resolved measurements so far have been mostly limited to small/biased samples (see above references) and do not permit the exploration of a wide parameter space for the study of the feedback phenomena.

A different strategy is given by the analysis of large area optical surveys, such as Sloan Digital Sky Survey (SDSS). In recent times, a number of studies have focused on the analysis of the kinematics of the [O III] $] 55007$ line ([O III] hereinafter) with the main goal of inferring the presence of ionized outflows (e.g. Bae \& Woo 2014; Concas et al. 2017; Komossa et al. 2008; Woo et al. 2016). By combining SDSS with multiwavelength data sets, several constraints on the properties and the effects of such outflows can be derived, at least in a statistical sense. For example, Mullaney et al. (2013), combining SDSS data with radio luminosity $\left(L_{1.4 \mathrm{GHz}}\right)$, found a strong correlation between $L_{1.4 \mathrm{GHz}}$ and the width of [O III], suggesting a connection between the presence of compact radio cores and the outflow phenomena (see also Zakamska et al. 2016; see Woo et al. 2016, for a different interpretation). Other recent studies related the presence of outflows with star formation activity using several diagnostics from Herschel and Spitzer data (Balmaverde et al. 2016; Wylezalek et al. 2016), which point to different conclusions regarding the role of AGN feedback (see also Mullaney et al. 2015). Despite some contradictory conclusions, all these works have shown that a large fraction of SDSS AGNs present signatures of outflows in their ionized phase, from $\sim 20-40 \%$ to $\sim 50$ $70 \%$ depending on whether the objects are type 2 or type 1 AGNs (Woo et al. 2016; Véron-Cetty et al. 2001). Moving to higher redshifts, Harrison et al. (2016) analysed a sample of $\sim 40$ X-ray selected objects at $z \sim 1$ and reported a fraction of outflows $\sim 50 \%$. Moreover, our recent works in the framework of XMMCOSMOS survey (Brusa et al. 2015, 2016; Cresci et al. 2015a; Perna et al. 2015a,b) have shown that the few ( $\sim 10$ sources) luminous obscured AGN at $z \sim 1.5$ with evidence of outflows collected so far, have relatively low X-ray $k_{\text {bol }}$ corrections (me$\operatorname{dian} L_{\mathrm{bol}} / L_{\mathrm{X}} \approx 10$ ). For comparison, more typical ratios of $\sim 40$ are found for sources at the same median bolometric luminosity (Lusso et al. 2012). A systematic study of outflow signatures in $\mathrm{X}$-ray selected samples is needed to validate a connection between outflow phenomena and X-ray loudness.

This work is part of a series of papers investigating the physical and demographic characterization of the AGN-galaxy system during the feedback phase. In this first paper we present a large sample of X-ray selected AGN at $z<0.8$, for which SDSS spectra are available. Similar to the analysis derived by previous works that combined optical spectroscopic analysis with radio and/or infrared wavelength bands, it extends the analysis for the first time to an X-ray selected large sample (>100 AGNs). We present the X-ray/SDSS sample selection procedure and the results obtained from relevant optical diagnostics. We focus on the incidence of outflows that can be derived by studying ionized and atomic features in optical spectra, which is crucial to better constrain the temporal lengths of the different AGN phases in the context of evolutionary models (e.g. Hopkins et al. 2008). We also derive the X-ray bolometric corrections for each AGN and test the role of X-ray activity in the context of the feedback phase.

The paper is organized as follows: in Sect. 2 we outline our sample selection procedure; in Sect. 3 we describe our multicomponent line fitting routine and the different emission line diagnostics. BPT diagrams are used to discriminate between star forming galaxies and AGNs among the X-ray selected sources. In Sect. 4 we estimate their nuclear properties. In Sects. 5 and 6 we derive the outflow fraction in the ionized and neutral phase, respectively, for different AGN subsamples. In Sect. 7 we test if a X-ray loudness is actually associated with outflow processes. Finally, we summarize our results and their implications in the last section (Sect. 8). A flat universe model with a Hubble constant of $H_{0}=72 \mathrm{~km} \mathrm{~s}^{-1} \mathrm{Mpc}^{-1}, \Omega_{\mathrm{M}}=0.27$ and $\Omega_{\lambda}=0.73$ is adopted.

\section{Sample}

We constructed our AGN sample by analysing SDSS data release 7 (DR7) spectra associated with X-ray emission taking advantage of previous studies in which the identification of the $\mathrm{X}$-ray detected sources with optical counterparts has been performed with accurate methods. In particular, we considered the following four studies:

- The catalogue compiled by Georgakakis \& Nandra (2011) obtained from the Serendipitous XMM Survey (Watson et al. 2009) in the area of the SDSS (XMM/SDSS), containing 2067 X-ray point sources with spectroscopic observations from the SDSS DR7 (Abazajian et al. 2009).

- The Jin et al. (2012) sample of 51 unobscured type 1 AGNs selected to have high-quality spectra from both XMMNewton and the SDSS DR7.

- The Wu et al. (2012) catalogue, whose 1034 objects are identified by matching the Swift pointings with SDSS DR5 quasar catalogue.

- The Trichas et al. (2013) catalogue, containing 617 matched sources from the Chandra Source Catalogue and the SDSS DR7 spectroscopic sample (CSC/SDSS) at $z<0.4$.

We selected the $z<0.8$ galaxies from this original sample of 3769 targets to include the [O III] emission line in the SDSS wavelength coverage. We selected all sources with signal-tonoise ratio $(\mathrm{S} / \mathrm{N})$ in the $[\mathrm{O} \mathrm{III}]$ region $>10$ i) to unveil and analyse the faint wings of the [O III] profile and ii) to exclude lineless galaxies, which are typically red galaxies with no sign of AGN activity. After a final check for possible duplicates in the catalogues, we obtained a X-ray/SDSS sample containing 624 unique objects.

We downloaded the SDSS spectra from the SDSS archive and reanalysed them using a multicomponent fitting routine detailed below. The top panel of Fig. 1 shows the [O III] luminosity plotted against redshift, colour-coded according to increasing nominal SDSS S/N of each spectrum.

$\mathrm{X}$-ray properties were obtained from available spectral analysis results published by Jin et al. (2012), Trichas et al. (2013), and $\mathrm{Wu}$ et al. (2012). For the Georgakakis \& Nandra (2011) sample, only non-corrected luminosities were available. We therefore extracted X-ray spectra for all the sources in this subsample from XMM archive, applying standard spectral fit procedures with a simple or double power-law model to correct the luminosity for moderate absorption (Lanzuisi et al. 2013).

The X-ray subsamples we used all come from heterogeneous X-ray observations and the flux limits differ significantly from observation to observation. However, the final coverage of the X-ray luminosity versus redshift plane obtained from the X-ray/SDSS sample (Fig. 1, lower panel) is similar to that of a multi-layer (wedding cake) survey, with a large-area, shallow layer, corresponding to typical flux limit of $f_{2-10 \mathrm{keV}} \sim 3 \times$ $10^{-14} \mathrm{erg} / \mathrm{cm}^{2} / \mathrm{s}$, and a much smaller area covered at deeper flux limits, down to $\sim 5 \times 10^{-16} \mathrm{erg} / \mathrm{cm}^{2} / \mathrm{s}$. 


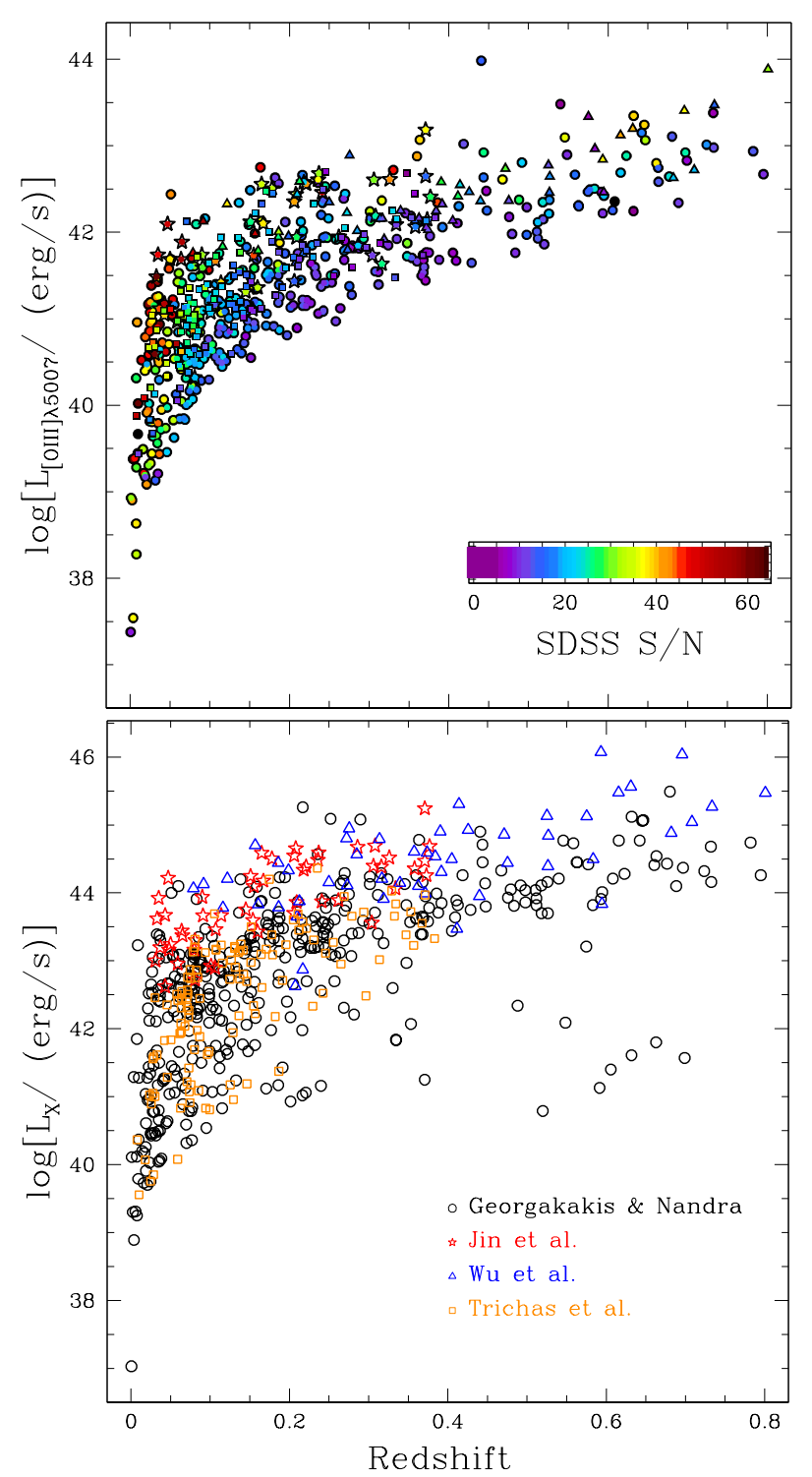

Fig. 1. Top panel: [O III] $\lambda 5007$ total luminosity as obtained by our spectral analysis vs. redshift for the 624 candidate AGNs coloured by nominal SDSS S/N, as labelled in the colour bar. Circles, stars, triangles, and squares refer to Georgakakis \& Nandra (2011), Jin et al. (2012), Wu et al. (2012), Trichas et al. (2013) subsamples, respectively, used to construct our X-ray/SDSS sample. Bottom panel: absorption corrected $\mathrm{X}$-ray luminosity vs. redshift for the same sample of targets, distinguishing between the different subsamples, as labelled in the figure.

Figure 2 (top panel) shows a schematic diagram of our selection process and summarizes the above-mentioned criteria and diagnostics described in the next sections.

\section{Spectral analysis}

\subsection{Multicomponent simultaneous spectral fit}

To characterize the nuclear accretion properties and determine kinematic and physical properties of the emitting gas, we proceeded by implementing the fitting prescriptions exposed in our previous studies (e.g. Brusa et al. 2015) to characterize the permitted Fe II ( $\lambda \lambda 4000-5400 \AA)$ emission features and He II $\lambda 4863$ line in the case of typical type 1 spectra.

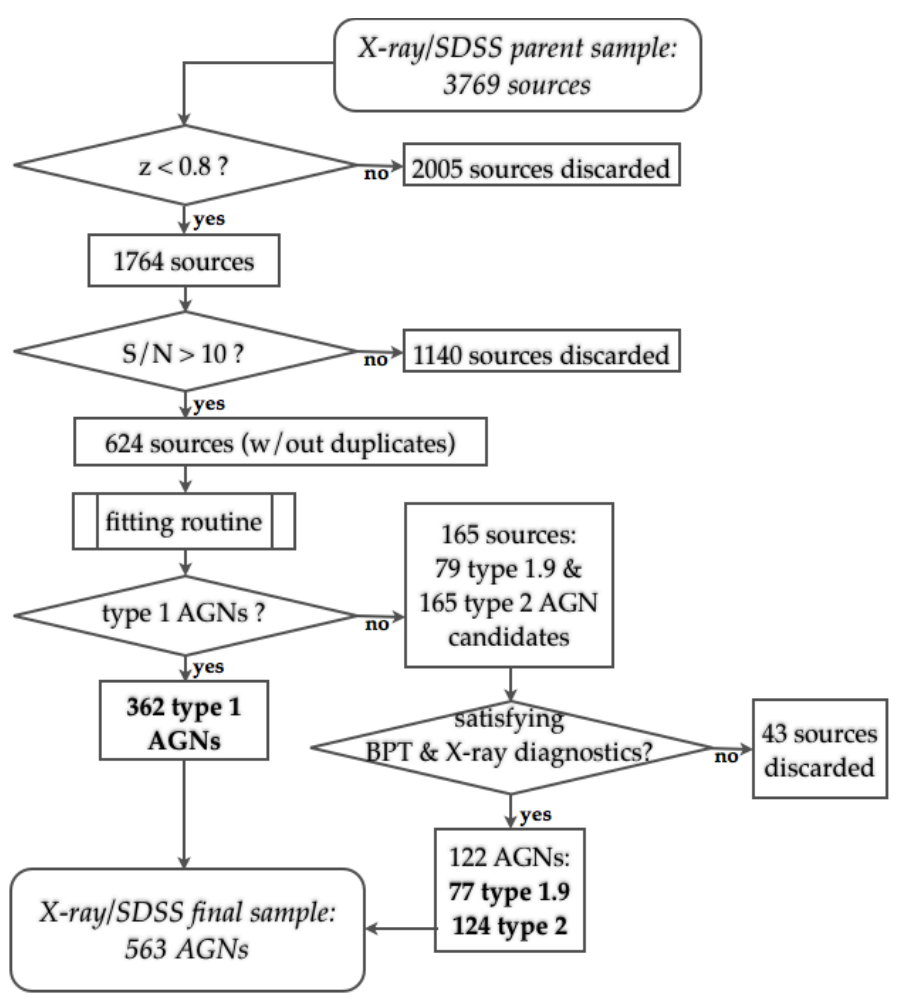

Fig. 2. Flow diagram representing the selection criteria (Sect. 2) and the diagnostics (Sect. 3) we used to define our X-ray SDSS sample.

We simultaneously fitted each of the most prominent emission lines, from the He II to [S II] doublet with four (at maximum) sets of Gaussian profiles as follows:

BC - One (two) broad component(s): three Gaussian (or Lorentzian) functions for the $\mathrm{He}$ II, $\mathrm{H} \beta$, and $\mathrm{H} \alpha$ BLR emission lines with a FWHM that is greater than $1000 \mathrm{~km} \mathrm{~s}^{-1}$. A second $\mathrm{BC}$ is added when $\mathrm{H} \alpha$ and $\mathrm{H} \beta$ BLR cannot be modelled with a single broad Gaussian profile (see e.g. Shen \& Kelly 2012).

NC - One narrow component: nine Gaussian lines, one for each emission line in the two regions of interest (i.e. He II, $\mathrm{H} \beta$, [O III] doublet, $\mathrm{H} \alpha$, [N II], and [S II] doublets) to account for the presence of unperturbed systemic emission (from the NLR or the host galaxy). The width of this kinematic component is set to be $\$ 550-600 \mathrm{~km} \mathrm{~s}^{-1}$.

OC - One (two, in an handful of cases) outflow component(s): eight Gaussian lines, one for each emission line (with the exclusion of the He II, which is usually faint and never well constrained) to account for the presence of outflowing ionized gas. No upper limits are fixed for the width of this kinematic component.

In addition, we used theoretical model templates of Kovačević et al. (2010) to reproduce Fe II emission when detected. Prior to the modelling of the emission lines, we estimated the local continuum by fitting a power law to the spectra at both sides of the regions using those wavelength ranges not affected by prominent features and/or bad sky-subtraction residuals (e.g. 4000-4050 and 5600-5650 for the $\mathrm{H} \beta$ region). For each set of Gaussian profiles, we constrained the wavelength separation between emission line within a given set of Gaussian profiles in accordance with atomic physics. This means that we constrained the velocity offset of the OC from the narrow (systemic) components to be the same for all the emission 
lines. Moreover, the relative flux of the two [N II] and [O III] components is fixed to 2.99 and [S II] flux ratio is required to be within the range $0.44<f(\lambda 6716) / f(\lambda 6731)<1.42$ (Osterbrock \& Ferland 2006).

The different ionization potentials and critical densities of NLR line features suggest a gas stratification with respect to the central SMBH (De Robertis \& Osterbrock 1986). In order to account for such an effect, the fitting procedure would need to consider a different line width for each emission feature within a given set of Gaussian lines. Our fitting procedure has proved to well reproduce all the analysed emission lines with the constraints mentioned above from low ionization emission lines, such as [S II] doublet (with IP $=10.4 \mathrm{eV}$ ), to high ionization lines (i.e. [O III] with IP $=35 \mathrm{eV}$; see also Lanzuisi et al. 2015). Indeed, even when different line widths were derived from low-to-median wavelength resolution data, their values were always consistent within the errors of the Gaussian fit (see De Robertis \& Osterbrock 1986; Wang \& Xu 2015). We therefore conclude that the spectral resolution of the spectra we analysed do not permit us to unveil a possible NLR stratification.

\subsection{Non-parametric measurements}

We used a non-parametric velocity estimator analysis (see e.g. Zakamska \& Greene 2014; Liu et al. 2013; Rupke \& Veilleux 2013a), for the [O III] emission line only, to derive the kinematic conditions of the ionized gas within the AGN NLRs.

We obtained on-parametric measurements by measuring velocity, $v$, at which a given fraction of the line flux is collected, using the cumulative flux function $F(v)=\int_{-\infty}^{v} F_{v}\left(v^{\prime}\right) \mathrm{d} v^{\prime}$. The position of $v=0$ of the cumulative flux is determined using the systemic redshifts derived from the NC of the simultaneous best-fit results. Following the prescription indicated by Zakamska \& Greene (2014), we estimated, for the total emission line best-fit profiles, the following parameters:

(i) The line width $W 80$, the width comprising $80 \%$ of the flux, which for a Gaussian profile is very close to the FWHM value. $W 80$ is defined as the difference between the velocity at $90 \%(v 90)$ and $10 \%(v 10)$ of the cumulative flux, respectively.

(ii) The maximum velocity parameter $V_{\max }$, defined as $v 02$ when blue prominent broad wings are present, or as $v 98$ when red wings, on the contrary, are present.

In contrast to $V_{\max }$, values of $W 80$ include only differences between velocities and do not depend on the accurate determination of the systemic velocity. A possible residual error in the determination of the systemic velocity, however, may produce a variation of at most few tens of $\mathrm{km} \mathrm{s}^{-1}$ in the $V_{\max }$ value, corresponding to variation of a few $\%$ for velocities higher than $500 \mathrm{~km} \mathrm{~s}^{-1}$ (see e.g. Fig. 8).

The best-fit line profile used to derive non-parametric estimates was taken from the simultaneous fitting procedure results. Such an approach is, in fact, less dependent on particular poor $\mathrm{S} / \mathrm{N}$ conditions in the vicinity of the emission line: the low $\mathrm{S} / \mathrm{N}$ regions around an individual emission feature, if fitted independently, may be wrongly interpreted as extended wings and modelled with a faint and broad Gaussian profile.

We fitted the Gaussian profiles using a Fortran code implementing the Minuit package (James \& Ross 1975). As a first step, we fitted all emission lines with minimum number of Gaussian components (i.e. $\mathrm{NC}$, and $\mathrm{NC}+\mathrm{BC}$ for broad line AGNs). The visual inspection of fit results allowed us to assess the necessity of a second fit with additional OC components when significant residuals are present. Then, we accepted the $\mathrm{NC}+\mathrm{OC}(+\mathrm{BC})$ fit decomposition if it resulted in a significant improvement in $\chi^{2}$ with $\Delta \chi^{2} \gtrsim 1000$ (reduced $\Delta \chi^{2} \gtrsim 1$ ), i.e. $\gtrsim 3 \sigma$. Our quality-of-fit criterion is justified by the large number of degrees of freedom $(\sim 1000)$ of simultaneous multicomponent fit. In fact, the number of parameters we used in $\mathrm{BC}+\mathrm{NC}$ or $\mathrm{BC}+\mathrm{NC}+\mathrm{OC}$ does not determine significant variations in $\chi^{2}$ distribution, and standard confidence intervals (Press et al. 1992) can be used to assess the possible improvement due to the addition of OC Gaussians.

In order to estimate errors associated with our measurements, we use Monte Carlo simulations. For each modelled spectrum, we collect the fit results of 30 mock spectra obtained from the best-fit final models (red curves in Figs. 3, 4) and adding Gaussian random noise (based on the standard deviation of the corresponding local continuum). When Fe II features were fitted, prior to the Monte Carlo simulations, we subtracted our best-fit Fe II template to minimize the degeneracy in the errors estimation. We calculated the errors by taking the range that contains $68.3 \%$ of values evaluated from the obtained distributions for each Gaussian parameter/non-parametric measurement. Finally, we note that since line profiles generally are non-Gaussian and much broader than the spectral resolution (see below), we do not correct the observed profiles for instrumental effects and report all values as measured.

\subsection{Spectral fit results}

Figure 3 plots five examples of different types of sources found in our sample: blue (a) and red (b) spectra of type 1 AGNs, in which Fe II emission and/or BC could be present; lowluminosity type 1 AGNs whose continuum are dominated by stellar emission (c); red spectra in which the same asymmetric profile is found in all optical emission lines and is therefore associated uniquely with OC without a clear sign of BLR Balmer emission (d); objects with double peaked emission lines that are modelled with two NC together with an OC (e).

We note that these AGN spectra often present significant Fe II emission. As described in Appendix A, such emission is strongly blended with BLR $\mathrm{H} \beta$ emission and the red wing of the [O III] line. As a result, to derive correct BLR profiles and nonparametric kinematics for the doubly ionized oxygen, an adequate modelling of the iron emission is required. We use theoretical model templates of Kovačević et al. (2010) to reproduce such emission. Two representative best-fit results showing strong blending with both $[\mathrm{O} \mathrm{III}]$ and $\mathrm{H} \beta$ lines are shown in Fig. A.1.

Through our own line fitting routine we found 441 broad line (BL) AGNs, comprising both type 1 and type 1.9 sources (showing $\mathrm{BC}$ in the $\mathrm{H} \alpha$ and not in higher order Balmer lines; see Fig. 3, panel c), and 165 type 2 AGN candidates. We excluded from any further analysis 7 [O III] double peaked galaxies ${ }^{1}$ (see e.g. Fig. 3, panel e; Fig. A.1, top panel), 5 red galaxies with high sky residuals at $\approx 5000$ Aresponsible for bad estimate of the $\mathrm{S} / \mathrm{N}$ in the [O III] region, and 6 galaxies with strong and complex stellar continuum in the proximity of $\mathrm{H} \alpha$ region.

1 Double peaked profiles could be associate both with bi-conical QSO winds and binary AGNs; SDSS spectra do not allow a separation between the two classes of objects (see discussion in Yuan et al. 2016, Sect. 3.2) and are therefore excluded. 


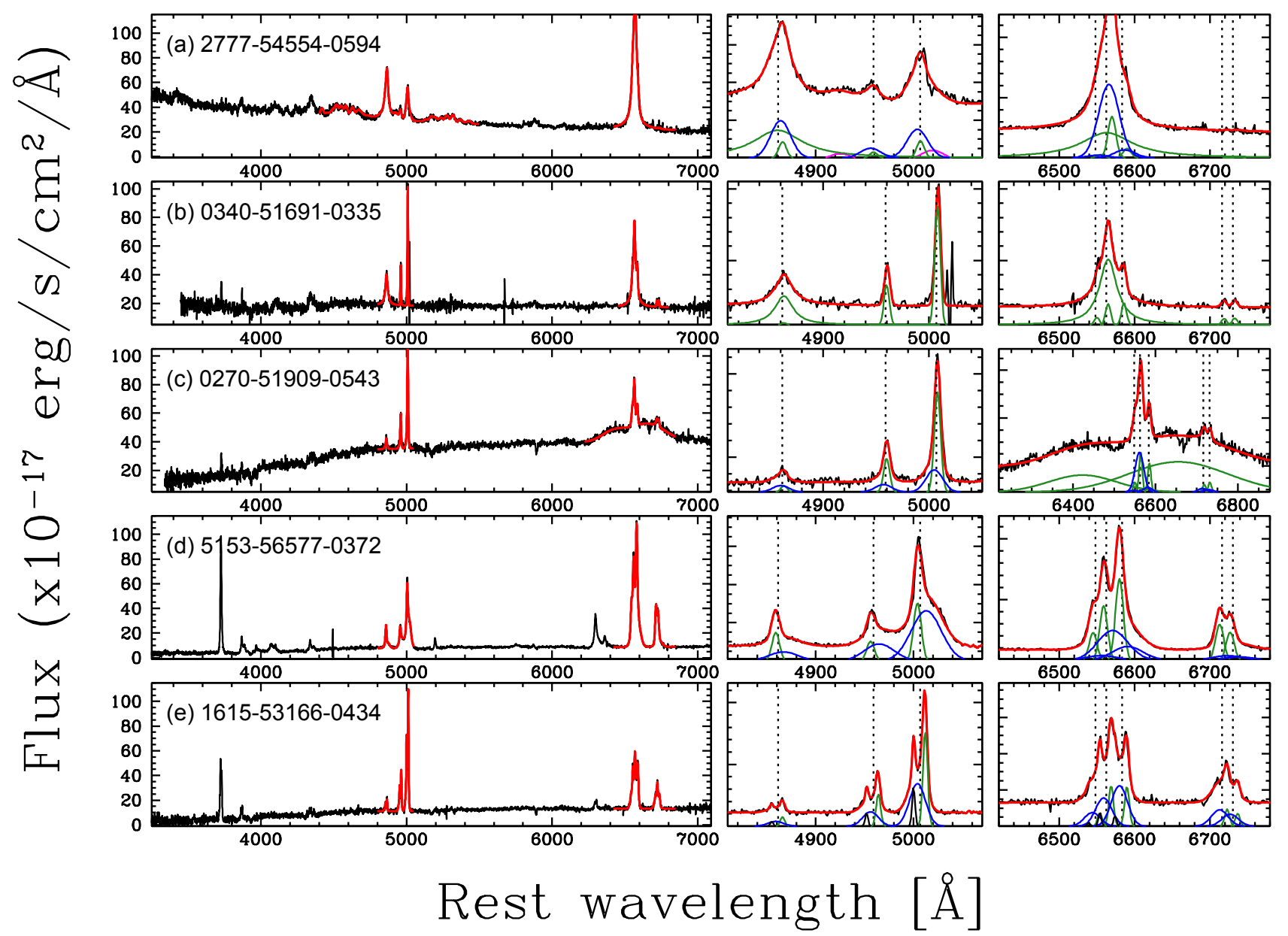

Fig. 3. Five examples of spectra illustrating our fitting method. For each object, we show on the left the spectrum (black curve) and the best-fit model (red curve). Central and right panels show the zoomed regions around the $\mathrm{H} \beta$ and $\mathrm{H} \alpha$ emission, respectively. The dashed vertical lines indicate the location of $\mathrm{H} \beta$, [O III] doublet, $\mathrm{H} \alpha$, [N II], and [S II] doublets. Best-fit NC and BC profiles are highlighted with green curves (panels a) to e)); OC and Fe II emission are shown with blue (panels a) to e)) and magenta (panel a)) curves, respectively. Finally, black Gaussian profiles (in panel e)) show a second set of NC used to fit doublet peaked galaxies. In the rows the spectra of various AGN types are shown. From top to bottom, the plots represent a) a blue spectrum of a type 1 AGN with broad BLR Lorentzian profiles, Fe II emission, and blue wings associated with outflows; b) a reddened type 1 AGN without evidence of outflow; c) a low-luminosity broad line AGN, whose continuum is dominated by stellar emission, in which we observe a complex BLR H $\alpha$ profile, modelled with two strong BC and no BLR H $\beta$ emission; d) a candidate type 2 AGN without evidence of BLR emission but with strong emission from outflowing gas detected for all the optical emission lines; and e) a candidate type 2 AGN with double peaked emission lines and an outflow component.

\subsection{BPT emission line diagnostics}

The presence of BLR emission represents an unambiguous indicator for AGN presence in the nucleus; however, some of our targets show concomitant stellar continuum and BLR features, indicating the presence of low-luminosity (or obscured) AGNs. Precisely, about $20 \%$ of our BL AGNs are type 1.9 sources. In order to confirm the nature of the AGN candidates and to discriminate between SF and AGN photoionized emission lines in faint type 1.9 and type 2 AGNs, we used the optical BPT diagnostic diagram (Baldwin et al. 1981) as a further tool to investigate the nature of the ionizing sources.

Prior to computing the line flux ratios, we fitted the stellar continuum using penalized pixel fitting (pPXF; Cappellari \& Emsellem 2004; Cappellari 2017) and corrected the Balmer line fluxes taking into account the stellar features from the pPXF best-fit model. In fact, underlying stellar absorption of the Balmer lines are not expected to be negligible in low-luminosity AGNs and could shape the emission line profiles. The pPXF routine is a programme developed by Cappellari et al. to extract the galaxy stellar kinematics (i.e. stellar velocity dispersion $\sigma_{*}$ ) from absorption-line spectra; to adopt the procedure for $\mathrm{BL}$ AGNs, a window of $1.2 \times 10^{4} \mathrm{~km} \mathrm{~s}^{-1}$ around the expected position of permitted emission lines is excluded from the fit. The pPXF method is able to well reproduce the continuum emission of $\sim 35 \%$ of the sample; we discarded all best-fit results associated with errors $>20 \%$ in velocity dispersion parameter. Figure 4 shows an example of a pPXF best-fit model for a type 1.9 AGN candidate (orange curve in the top panel). In the bottom panels we show the results obtained from the multicomponent simultaneous fit (red curves) and, in the insets, the excess in the Balmer emission lines found after the correction for the pPXF best-fit model. We found that the Balmer stellar absorption features can actually determine underestimates in the fluxes with median values of $\approx 5 \%$ and $\approx 20 \%$ of the $\mathrm{H} \alpha$ and $\mathrm{H} \beta$, respectively. As expected, such absorption features affect only the narrow emission components.

Figure 5 shows the BPT diagrams obtained from our spectroscopic analysis, after the correction from stellar features, for both NC (left) and OC (right). The lines drawn in the diagrams correspond to the theoretical curve (at $z=0$ ) used to separate 


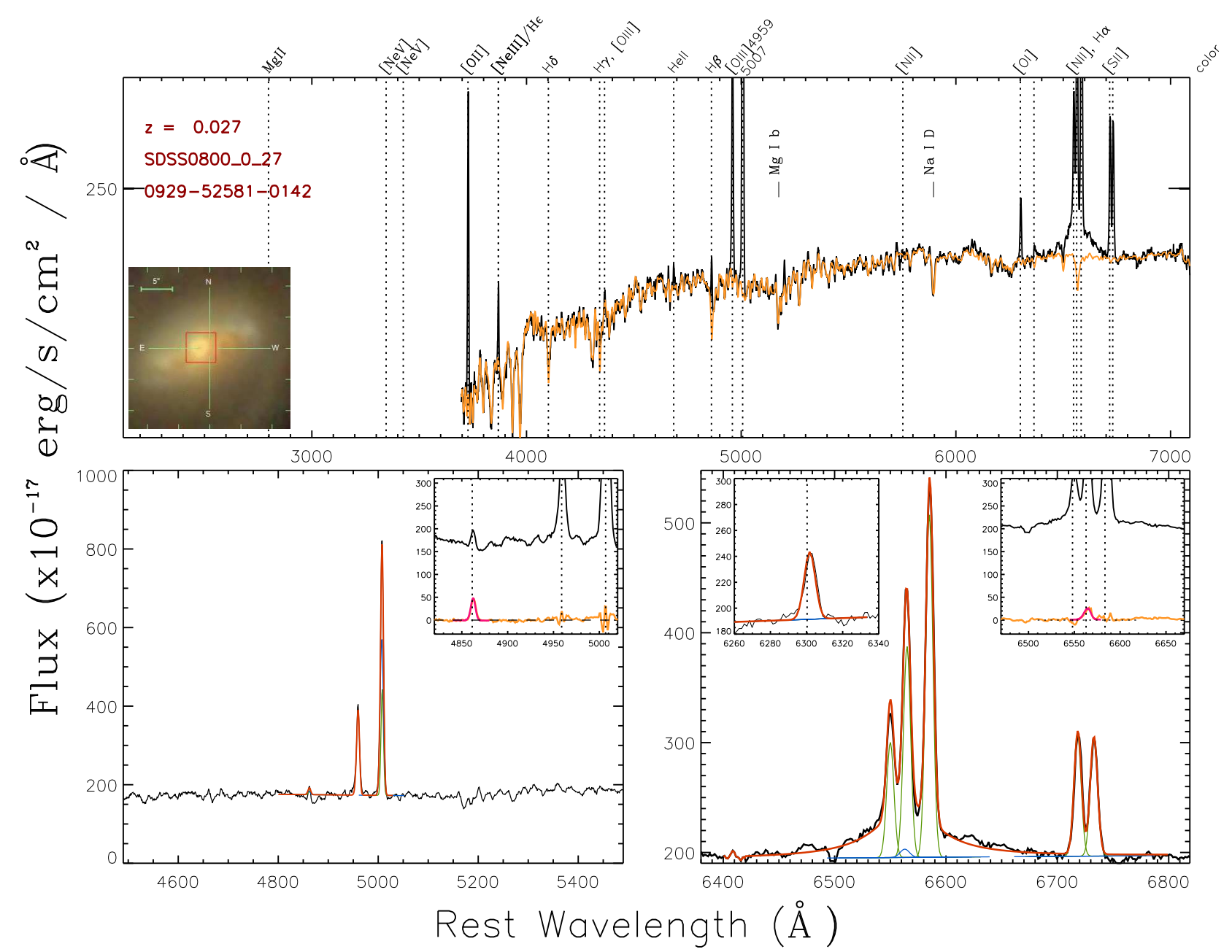

Fig. 4. Top panels: pPXF best-fit model results for a type 1.9 AGN (orange curve) superimposed on the rest-frame SDSS spectrum. All the prominent emission lines and analysed features are highlighted with vertical dotted lines; the positions of $\mathrm{Mg}$ I b and Na I D absorption lines are also indicated. The inset on the left shows the SDSS colour-composite cut-out of the galaxy with a red square indicating the spatial region from which the $3^{\prime \prime}$ spectrum was obtained. Bottom panels: fit results from the multicomponent simultaneous fit in the $\mathrm{H} \beta$-[O III] (left) and $\mathrm{H} \alpha$ [NII] (right) regions. Green and blue curves show the NC and OC Gaussian profiles, while the red lines represent the total best-fit profile. The insets on the right of each panel show the excess in the Balmer emission lines found after the correction for the pPXF best-fit model (the orange curves represent the difference between the SDSS spectrum and pPXF and simultaneous fit models). The original spectrum is also shown for a qualitative evaluation of the contribution of stellar absorption feature in the observed emission line. Magenta Gaussian profiles represent best-fit results associated with significant $(>5 \sigma)$ residuals. The insets on the left of the $\mathrm{H} \alpha+[\mathrm{N} \mathrm{II}]$ panels show the best-fit models of [O I] $\lambda 6300$.

purely SF galaxies from galaxies containing AGN (Eq. (1) of Kewley et al. 2013).

The use of BPT diagrams for both NC and OC is justified by recent results obtained with spatially resolved spectroscopy, which probes the different locations and ionization conditions of unperturbed and outflowing gas (e.g. Arribas et al. 2014; Cresci et al. 2015a,b; Kakkad et al. 2016; McElroy et al. 2015; Perna et al. 2015a,b; Westmoquette et al. 2012).

For almost all the sources, the systemic NC and the OC are consistent with an AGN classification. Outflow component measurements are more scattered because of their associated lower intensities: this determines an important degeneracy in the fit results, in particular for type 1 AGNs (blue circles) for which a higher number of components must be taken into account (namely, $\mathrm{NC}$, OC, and $\mathrm{BCs}$ ).

Because of the mentioned arguments, we excluded from the following analysis 43 targets (indicated with red crosses in the figure) using only NC flux ratios. For these sources, the SF nature highlighted by the BPT diagrams, has been confirmed by the concomitance of red spectra and low X-ray luminosities (i.e.
$<10^{42} \mathrm{erg} / \mathrm{s}$ in the $2-10 \mathrm{keV}$ band). The exclusion of few targets above the theoretical transitional curve is due to a conservative approach that takes into account, first, a possible stellar absorption feature contribution in those sources for which low $\mathrm{S} / \mathrm{N}$ spectra do not allow stellar features modelling, but for which we expect some contribution (i.e. type 1.9 and type 2 sources). In order to account for this effect, we derive representative shifts in the BPT diagram due to possible underestimates in Balmer flux estimate, assuming that all $\mathrm{H} \alpha$ and $\mathrm{H} \beta$ fluxes are increased by a factor of $3 \times$ the median $\mathrm{pPXF}$ corrections mentioned above. These "corrections" correspond to a downward shift of $\approx 0.2$ in $\log ([\mathrm{O} \mathrm{III}] / \mathrm{H} \beta)$ and a left shift of $\approx 0.1$ in $\log ([\mathrm{N} \mathrm{II}] / \mathrm{H} \alpha)$. Second, this approach also takes into account error bars associated with each source, due to strong degeneracy in the fitting procedures when low $\mathrm{S} / \mathrm{N}$ spectra are analysed.

About $14 \%$ of our targets have $z>0.4$ and their $\mathrm{H} \alpha$ region is not covered by the SDSS spectra. For these targets it is therefore not possible to use the BPT diagnostic. However, they are generally associated with blue spectra and $95 \%$ of them show unambiguous BLR $\mathrm{H} \beta$ emission. For the remaining 5\% (four targets) 


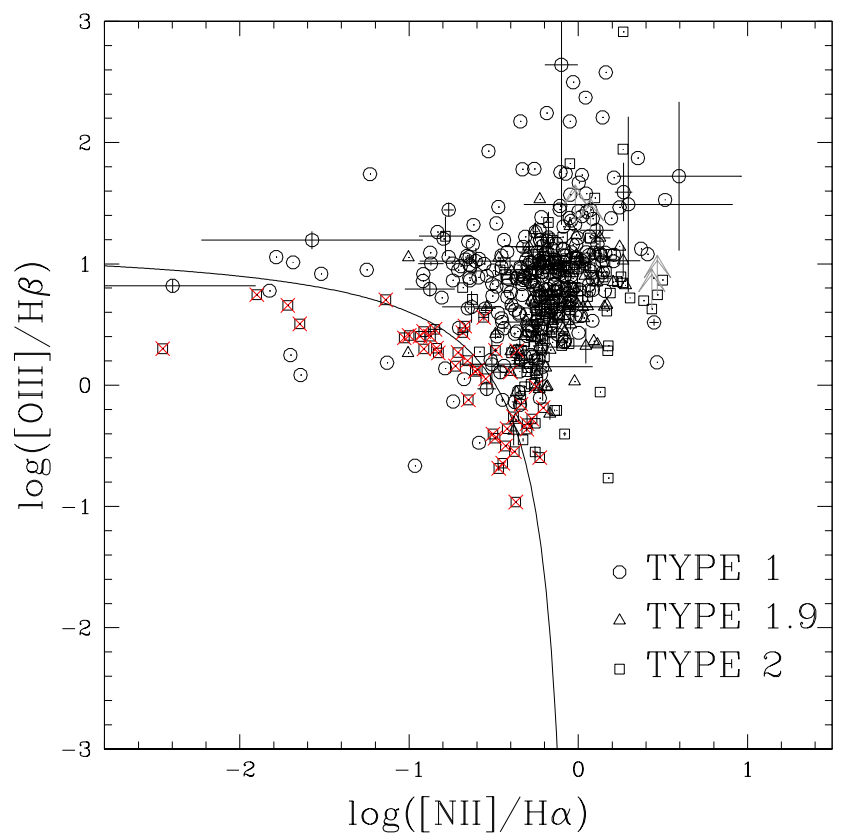

(a)

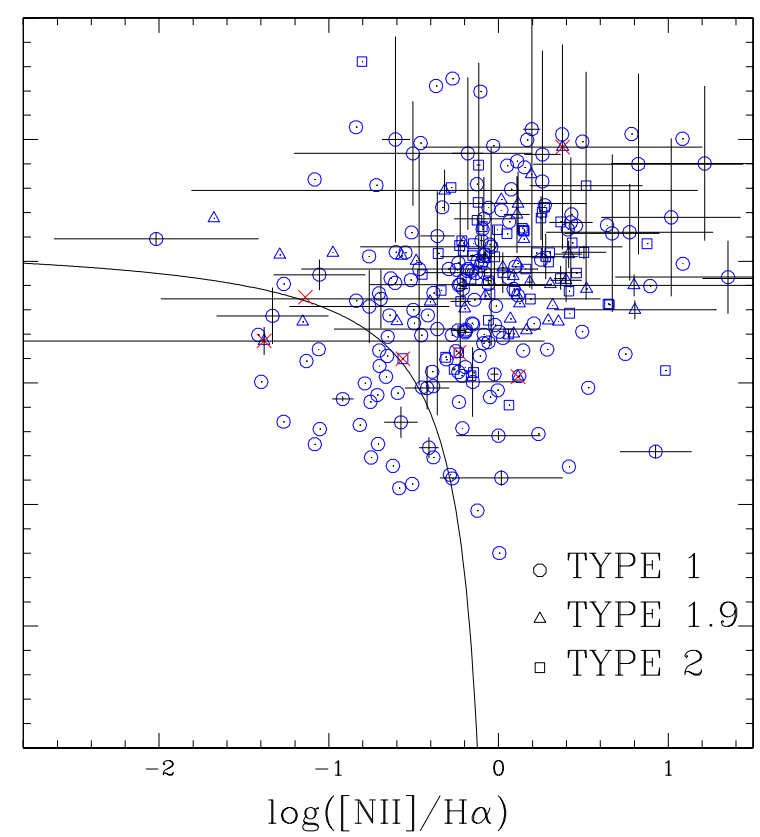

(b)

Fig. 5. BPT diagrams - standard diagnostic diagram showing the classification scheme by Kewley et al. (2013). The lines drawn in the diagram correspond to the theoretical redshift-dependent curves at $z=0$ used to separate purely SF galaxies from galaxies containing AGN. Black (left panel) and blue (right panel) symbols correspond to the systemic NC and outflow OC flux ratios, respectively. Representative error bars are shown only for a small fraction of targets. Circles, squares, and triangles denote Type 1, type 2, and type 1.9 AGNs, respectively. Red crosses highlight the SF galaxies discarded from the sample, using the NC flux ratios (see text for details). Grey upper arrows represent lower limits due to an undetected $\mathrm{H} \beta$ emission line.

we observed $[\mathrm{O} \mathrm{III}] / \mathrm{H} \beta$ consistent with the average value observed for the entire sample $(\log [\mathrm{O} \mathrm{III}] / \mathrm{H} \beta \sim 1)$, for both $\mathrm{NC}$ and, when present, OC. We therefore confirm the AGN nature for all the $z>0.4$ targets.

Summarizing, thanks to the BPT diagnostic coupled with a visual inspection of the spectra and the available X-ray analysis, we obtain a final sample of 563 AGNs (362 type 1, 77 type 1.9, and 124 type 2 ).

\section{Nuclear properties}

We used the single-epoch (SE) technique to determine the $\mathrm{BH}$ masses of our subsample of BL AGNs. This technique combines the virial theorem with the BLR radius - luminosity relation (see e.g. Kaspi et al. 2005; Bentz et al. 2013; Saturni et al. 2016), allowing an estimate of the black hole mass from measurements of the BLR line width and AGN luminosity. Different empirical relations have been calibrated in recent years (e.g. Vestergaard \& Peterson 2006; Shen et al. 2011; Bongiorno et al. 2014) for various BLR line width and AGN luminosity indicators. Here we adopted the $\mathrm{SE}$ relations calibrated by Greene \& Ho (2005),

$$
\begin{aligned}
& \log \left(\frac{M_{\mathrm{BH}, \mathrm{SE}}}{M_{\odot}}\right)=6.30_{-0.07}^{+0.08} \\
& +(0.55 \pm 0.02) \log \left(\frac{L(\mathrm{H} \alpha)}{10^{42} \mathrm{erg} / \mathrm{s}}\right)+(2.06 \pm 0.06) \log \left(\frac{F W H M}{10^{3} \mathrm{~km} \mathrm{~s}^{-1}}\right) \\
& \log \left(\frac{M_{\mathrm{BH}, \mathrm{SE}}}{M_{\odot}}\right)=6.56_{-0.03}^{+0.02} \\
& +(0.56 \pm 0.02) \log \left(\frac{L(\mathrm{H} \beta)}{10^{42} \mathrm{erg} / \mathrm{s}}\right)+2 \log \left(\frac{F W H M}{10^{3} \mathrm{~km} \mathrm{~s}^{-1}}\right)
\end{aligned}
$$

These relations replace the $\lambda L_{\lambda}$ at $5100 \AA(L(5100 \AA)$ hereinafter) usually associated with SE relations calibrated on Balmer lines (Shen et al. 2011), with their BLR luminosities, and are based on the tight correlations found between Balmer and continuum luminosities in type 1 AGNs with strong blue thermal nuclear continuum (see Greene \& Ho 2005, Fig. 2). We adopted such relations to mitigate the effects of host galaxy contamination at $5100 \AA$ in our sample. Figure 6 shows the Balmer luminosities against the $5100 \AA$ continuum luminosity for our BL AGNs. The two panels show a significant displacement from the Greene and Ho relations at lower Balmer luminosities $\left(L(\mathrm{H} \alpha)\right.$ and $\left.L(\mathrm{H} \beta) \lesssim 10^{42} \mathrm{erg} / \mathrm{s}\right)$, owing to the enhancement in the continuum luminosity $L(5100 \AA)$, which is strongly contaminated by the host galaxy emission.

In order to derive the black hole masses, we adopted the FWHM measured from the best-fit model of the BLR profile as line width (see e.g. Shen et al. 2011). Such procedure takes into account the additional multiple Gaussian (and/or Lorentzian) components required to reproduce peculiar broad $\mathrm{H} \alpha$ and $\mathrm{H} \beta$ BLR line profiles (see e.g. Fig. 3, panel c). With these assumptions on AGN luminosity and FWHM, we derived black hole masses in the typical range $10^{6}-10^{9} M_{\odot}$.

We also estimate AGN bolometric luminosities. To compute an X-ray bolometric correction and test, in a statistical way, the results we obtained in our previous works (e.g. Brusa et al. 2016), we need a bolometric luminosity estimator that is independent from X-ray emission. In fact, contrary to the small samples at higher redshifts carefully selected in the COSMOS field, and for which we could derive all the critical nuclear AGN properties through spectral energy distribution (SED) fitting procedures, this SDSS sample does not allow such an accurate approach. Hence, we should refer to the 


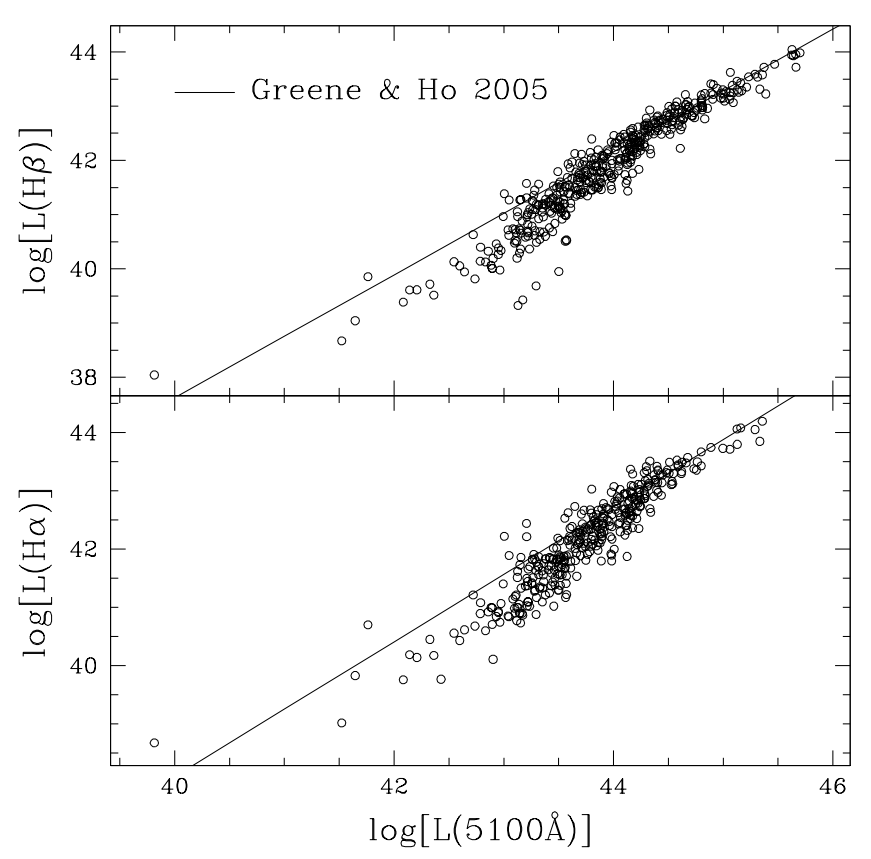

Fig. 6. Correlations of $L_{\mathrm{H} \alpha}-$ (bottom panel) and $L(\mathrm{H} \beta)-L(5100 \AA)$ (bottom). The solid lines represent the Greene \& Ho (2005) correlations obtained from a sample of type 1 AGN with low galaxy contribution in the optical continuum emission. A significant displacement from such relations is found for our low-luminosity AGN subsample, in which the continuum emission at $5100 \AA$ is dominated by host galaxy emission.

SDSS spectral information. The unperturbed NC of the [O III] line is usually referred as a good tracer of the AGN luminosity (e.g. Panessa et al. 2006; Jin et al. 2012; while the OC emission may be due to different processes, such as shocks). However, we excluded the use of [O III] line to reduce possible spurious correlations in our analysis (e.g. the outflow velocity estimators depend on the NC flux of the [O III] line). Instead, we chose to use the $5100 \AA$ indicator, taking advantage of the above-mentioned BLR-continuum luminosity relations to "correct" the observed $L(5100 \AA)$ in the low-luminosity regime, and applying the luminosity-dependent bolometric correction ${ }^{2}$, $b(5100 \AA)=53-\log [L(5100 \AA)]$, which is presented by Netzer \& Trakhtenbrot $(2014)^{3}$.

\section{Incidence of ionized outflows}

In order to discriminate between gravitational and outflow processes, we chose a conservative velocity threshold $V_{\max }=$ $650 \mathrm{~km} \mathrm{~s}^{-1}$. This value corresponds to the maximum velocity derived for a Gaussian profile with a velocity dispersion of $340 \mathrm{~km} \mathrm{~s}^{-1}$, and was chosen considering that $95 \%$ of massive BOSS galaxies below $z=0.8$ have velocity dispersions lower than this value (Thomas et al. 2013).

Critical broadening in forbidden emission lines can also be originated by merging events or inflowing gas. We stress however that maximum velocities higher than $\sim 600 \mathrm{~km} \mathrm{~s}^{-1}$ are not common even in sub-millimeter galaxies at $z \sim 2$, where we expect to observe ongoing mergers (see e.g. Collet et al. 2016),

\footnotetext{
2 Bolometric corrections convert a luminosity at a specific wavelength to $L_{\mathrm{bol}}$, considering typical AGN SED.

3 Unperturbed NC doubly ionized oxygen and host-galaxycontribution corrected continuum luminosities are well correlated (Spearman ratio of 0.85 with null hypothesis probability $<10^{-5}$ ).
}

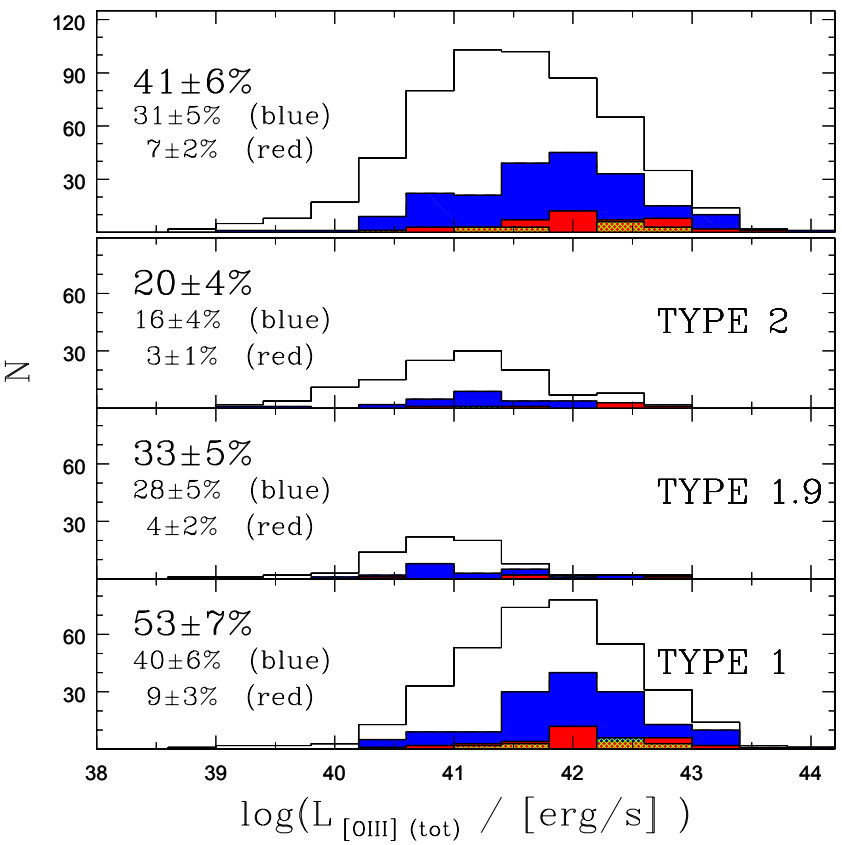

Fig. 7. Distributions of sources with ionized outflows as a function of the [O III] luminosity. The top panel shows the velocity distribution for approaching, receding, and symmetric outflows represented with blue, red, and green histograms, respectively. The lower panels show same distributions for subsamples defined on the basis of the AGN type. For each panel, the fraction of AGNs with outflow is highlighted, together with the fraction of receding and approaching outflows.

or in inflowing gas (e.g. Bouché et al. 2016, and references therein).

We assume that $V_{\max }$ is representative of the outflow velocity (assumed to be constant with radius). From a geometrical point of view, we consider that $V_{\max }$ is proper of emitting gas close to our line of sight, and that all lower velocities observed in oxygen line profile are due to projection effects (see Cresci et al. 2015a). These assumptions, generally adopted in literature (e.g. Brusa et al. 2016; Cano-Díaz et al. 2012; Kakkad et al. 2016), are required because of the still unsettled geometry of the outflow (Carniani et al. 2015; Cicone et al. 2014).

Figure 7 (top panel) shows the distribution of sources against [O III] luminosity for the full sample and for three subsamples defined on the basis of the presence/absence of blue or red prominent wings in the [O III] profile. We found signature of outflows in $\approx 41 \%$ of AGNs, of which $31 \%$ and $7 \%$ have blue and red prominent wings, respectively, while only $3 \%$ show symmetric profiles. The lower panels in the figure show the distributions for a given spectral type, i.e. splitting the sample in type 2, type 1.9, and type 1 AGNs: the fraction of outflows increases going from type 2 to 1 up to over $50 \%$ in type 1 AGNs. In particular, the relative fraction of blue/red outflows are roughly similar in each spectral type: the fraction of incoming outflows are always $\sim 5$ times that of receding outflows. In the context of the unified model, in type 1 AGNs the torus structure should force the outflowing gas towards our direction, i.e. we should be able to observe more sources with blue outflows. On the contrary, in type 2 AGNs, the torus axis should be perpendicular to our line of sight and the ejected material should emerge along that direction; the projected outflow velocities in this case are smaller, easily explaining the lower number of sources with outflows in type 2 AGNs. Naivily, one would expect to observe a larger fraction of symmetric outflows in type 2 AGNs. However, the dust 


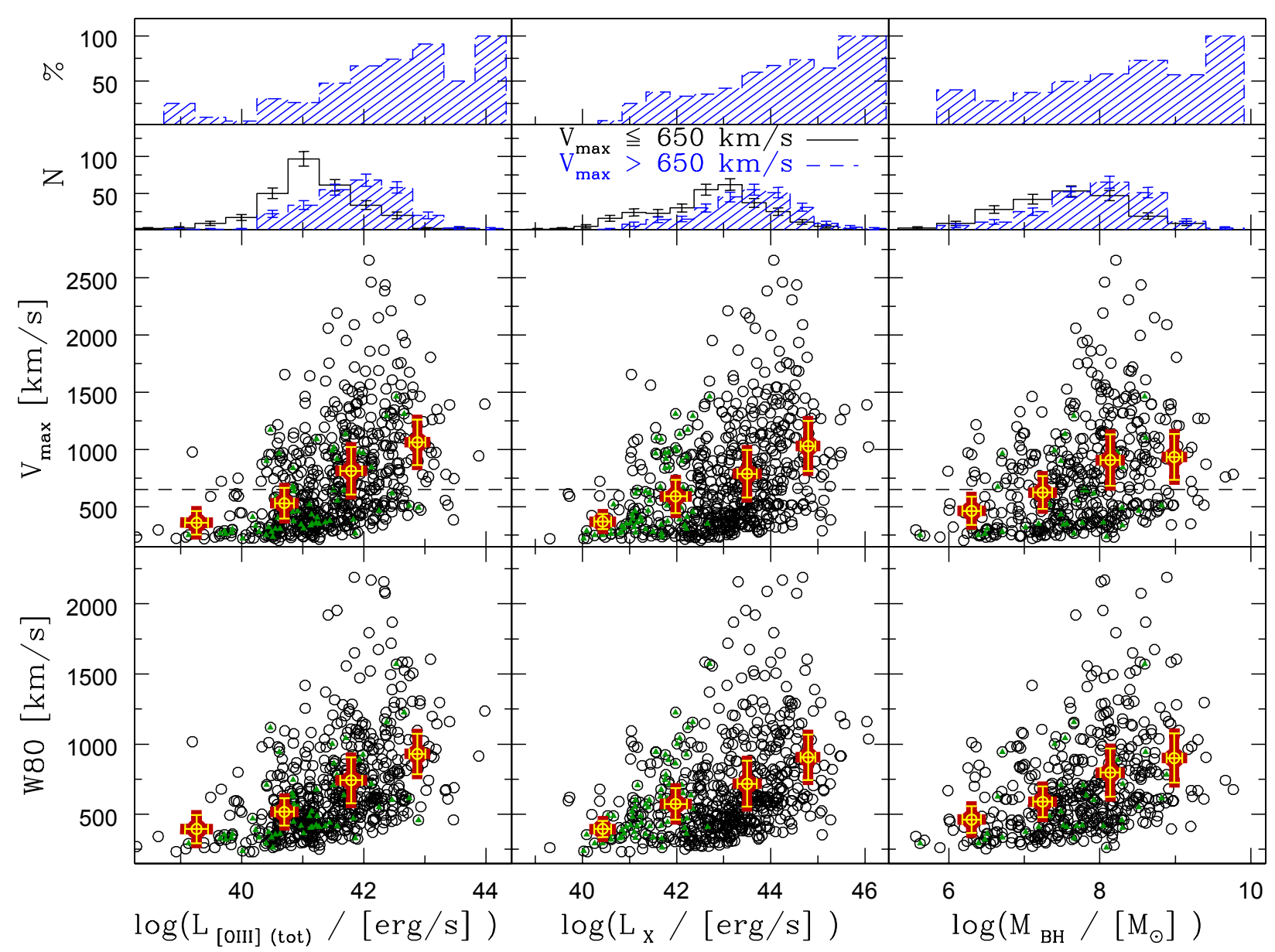

Fig. 8. Central and bottom panels: non-parametric velocity $W 80$ and $V_{\max }$ estimators as a function of [O III] (left) and intrinsic $2-10 \mathrm{keV}$ X-ray (central) luminosity, as well as a function of the black hole mass for the BL AGN subsample. Red/yellow dots represent average velocity in bin of luminosity; bars indicate uncertainties of the averages. Green dots indicate faint X-ray sources for which the intrinsic $L_{\mathrm{X}}$ has been inferred using HR ratio corrections (see text). Top panels: fraction of AGNs with $V_{\max }>650 \mathrm{~km} \mathrm{~s}^{-1}$ (blue dashed line; first panel from the top) and distributions of $V_{\max }<650 \mathrm{~km} \mathrm{~s}^{-1}$ (black line) and $V_{\max }>650 \mathrm{~km} \mathrm{~s}^{-1}$ (blue dashed line; second panel from the top) as a function of [O III] (left), X-ray (central) luminosity, and $M_{\mathrm{BH}}($ right $)$.

distribution in the disk and the inclination of the host galaxy with respect to the line of sight is probably enough to obscure a large fraction of the flux from the receding outflow, regardless of the AGN spectral classification (see e.g. Fischer et al. 2013; Müller-Sánchez et al. 2011; Bae \& Woo 2014).

Figure 8 shows the distributions of the two velocity estimators against the total [O III] (on the left) luminosities, the intrinsic X-ray 2-10 keV (centre) luminosities, and the black hole masses for the BL AGN subsample (right panels). We discuss here the major results for each pair of indicators:

$V_{\text {out }}$ versus $L_{[\mathrm{O} \text { III] }}$ : both non-parametric estimators show a positive correlation with increasing [O III] luminosity: moving from lower to higher luminosities, an increasing number of targets exhibit higher velocities. A clear trend appears when we consider the average velocities in bin of luminosity (redyellow points in the lower and central panels). The same positive trend also appears when we consider median instead of average values, or when we combine our results with those available from literature (see Appendix B). We also investigate how the fraction of AGNs with/without outflows changes as a function of the $L_{[\mathrm{O} \text { III] }}$ (per luminosity bins; first row in the left panel). We found that above $L_{[\mathrm{O} \text { III }]} \approx 10^{42} \mathrm{erg} / \mathrm{s}$ the fraction of AGNs with outflows becomes $>50 \%$, as also highlighted in the second row first (left panel), where we show the number of AGNs with $V_{\max }$ above/below the velocity threshold value.
Assuming a bolometric correction of $\sim 3 \times 10^{3}$ for the [O III] line (Heckman et al. 2004), this corresponds to a bolometric luminosity of $\approx 10^{45} \mathrm{erg} / \mathrm{s}$, which is consistent with the luminosity threshold proposed by Veilleux et al. (2013), obtained by studying the incidence of molecular outflows in ULIRGs hosting AGNs, and by Zakamska \& Greene (2014) and Woo et al. (2016), derived by analysing ionized outflows in obscured and type 2 QSOs.

$V_{\text {out }}$ vs. $L_{\mathrm{X}}$ : the same results are found when we consider the $\mathrm{X}$-ray luminosities (central panels): a clear trend with increasing $L_{\mathrm{X}}$ is observed, both with $W 80$ and $V_{\max }$ measures. Also in this case, the fraction of sources with outflows is higher in the high-luminosity regime (upper panel). We underline that this is the first time we observe such correlations between X-ray activity and outflow effects in a large sample, confirming the results obtained on a smaller sample of $z \sim 0.6-1.6$ by Harrison et al. (2016) and based only on two luminosity bins (see their Fig. 8). In the low-luminosity regime, a small group of sources are shown with green symbols. For these sources we cannot derive the intrinsic X-ray luminosity from proper X-ray spectral analysis because they are associated with poor detections (i.e. $<100$ counts in the $2-10 \mathrm{keV}$ band). For these sources we show therefore the X-ray luminosities as inferred from the hardness ratio (HR) correction (following Merloni et al. 2014). Because a large percentage of these sources are associated with high 
HR, we suggest that, if properly corrected for extinction, these sources could even reinforce the correlation between X-ray luminosity and outflow velocity.

$V_{\text {out }}$ vs. $M_{\mathrm{BH}}$ : we also found the same positive correlations in the right panels. These trends are expected since the $M_{\mathrm{BH}}$ is derived from Eq. (1), i.e. proportional to a third indicator of the bolometric luminosity, the BLR Balmer emission. As for [O III] and X-ray luminosities, here we find a black hole mass threshold of $10^{8} M_{\odot}$. We stress that this result allows an interesting confirmation of the evolutionary model predictions (see King \& Pounds 2015): comparing the gas bulge binding energy of a typical massive galaxy of $M_{\text {bulge }} \sim 10^{11} M_{\odot}$, stellar velocity dispersion $\sigma_{*} \sim 300 \mathrm{~km} \mathrm{~s}^{-1}$ and a gas fraction $f_{\text {gas }}=0.162$ (the cosmological mean value; Planck Collaboration XVI 2014),

$E_{\text {bulge }} \sim f_{\text {gas }} M_{\text {bulge }} \sigma_{*}^{2} \approx 10^{59} \mathrm{erg}$,

with the predicted outflow energy computed under the assumption of a small coupling factor with the released SMBH energy $(\approx 1 \%$, as we actually found in AGN-driven outflows; see Carniani et al. 2015; Fiore et al. 2017), and an Eddington accreting mass rate,

$E_{\text {out }} \sim 0.01 \times E_{\mathrm{BH}} \approx 10^{51}\left(M_{\mathrm{BH}} / M_{\odot}\right) \mathrm{erg}$,

we find that only when the SMBH reaches a $M_{\mathrm{BH}} \sim 10^{8} M_{\odot}$ it is powerful enough to release such energy in the form of powerful outflows. Of course, the large uncertainties in deriving the $M_{\mathrm{BH}}$ (see discussion in Shen et al. 2011), the unknown host galaxy properties, and the assumed Eddington accretion onto the $\mathrm{SMBH}^{4}$ must be taken into account. For all these reasons, our results represent a mild confirmation of theoretical predictions.

A possible criticism of these $V_{\text {out }}-$ AGN power relations is that when $\left.L_{[\mathrm{O}} \mathrm{III}\right]$ is small, it is more difficult to detect and model faint wings associated with outflows. However, the sources associated with smallest luminosities are also those with smallest $z$ and highest $\mathrm{S} / \mathrm{N}$, as highlighted in Fig. 1 (see also the detailed discussion in Appendix C). Therefore, we can conclude that, overall, all these correlations show an increasing incidence of outflow processes with AGN power. We used the Spearman rank (SR) correlation coefficient to derive the significance of the observed trends. We found coefficients of $\approx 0.5-0.7$ with probabilities of $\ll 0.001$ for the correlation being observed by chance. Such correlations also explain the observed fractions of outflows found in different spectroscopic AGN types with a higher incidence in type 1 rather than in type 2 sources because the former are characterized, on average, by higher luminosities (see Fig. 7).

\section{Incidence of neutral outflows - Mg I b - Na I D diagnostic}

The Na I D $\lambda \lambda 5890,5896$ absorption features can be used to directly probe the neutral phase of outflowing gas (e.g. Rupke et al. 2005a; Villar-Martín et al. 2014). Figure 4 shows a spectrum with a strong sodium line that is well reproduced by the pPXF procedure, i.e. through stellar absorption. This is because both stellar and interstellar absorption could be at the origin of the

\footnotetext{
$4 \mathrm{We}$ are able to estimate the Eddington ratio $\lambda_{\mathrm{Edd}}=L_{\mathrm{bol}} / L_{\mathrm{Edd}} \propto$ $L_{\mathrm{bol}} / M_{\mathrm{BH}}$ for our sample of BL AGNs from the derived bolometric luminosities and black hole masses. Both sources with and without outflows are characterized by average Eddington ratio of $\approx 0.1$ with a possible mild evidence of higher $\lambda_{\mathrm{Edd}}$ in those sources with signatures of outflows. However, we avoid any significant conclusions because of the large uncertainties in deriving $\lambda_{\text {Edd }}$ and to AGN flickering considerations (e.g. Schawinski et al. 2015).
}

line. Therefore, we need to discriminate between the two contributions to study the neutral outflowing gas component. A simple diagnostic used to unveil the presence of neutral gas in the ISM is comparing the $\mathrm{EW}$ of $\mathrm{Na} \mathrm{I} \mathrm{D}$ with that of $\mathrm{Mg} \mathrm{I}$ b triplet at 5167, 5173 and $5184 \AA$, which is of pure stellar origin. Similar mechanisms by which sodium and magnesium are created in stars explain the simple relation $E W(\mathrm{Na}$ I D $)=0.75 \times E W(\mathrm{Mg}$ I b) (Heckman et al. 2000; Rupke et al. 2005a; Villar-Martín et al. 2014); any deviation from that to higher $\mathrm{EW}(\mathrm{Na} \mathrm{I} \mathrm{D})$ is therefore interpreted as due to the presence of interstellar absorption in the $\mathrm{Na} I \mathrm{D}$.

For the sources in our sample we derive a first order estimate for EW(Mg I b) from the best-fit pPXF profile. The EW of $\mathrm{Na} I \mathrm{D}$ is instead derived by modelling the spectra around the absorption feature. He I $\lambda 5876$ emission and $\mathrm{Na}$ I D absorption are fitted by constraining the central wavelengths and FWHMs using the simultaneous fit results. The diagnostic comparing $\mathrm{Mg} \mathrm{Ib}$ and $\mathrm{Na}$ ID EWs is shown in Fig. 9 (left). The majority of AGNs shows $\mathrm{Na}$ I D dominated by stellar contribution (i.e. they lie on the above-mentioned relation, shown in the figure as a dashed line), while only a small fraction of objects is located well below the correlation. When investigating the subsamples separately with and without ionized [O III] outflows (blue and black symbols in the figure, respectively), we do not find any difference in their location on the above-mentioned diagnostic.

Rupke et al. (2005a) found that the majority (80\%) of the galaxies below the relation $E W(\mathrm{Na}$ I D $)=3 \times E W(\mathrm{Mg}$ I b) have neutral outflows and proposed that objects in this region undergo a starburst-driven outflow episode. The solid line in the diagram shows the relation reported by Rupke et al. (2005a). Despite the high fraction of outflows found in ionized gas, this plot shows only one target below the Rupke et al. relation. This source, Mkn 848, is the only one for which we detect a neutral outflow, according to this diagnostic diagram.

In Fig. 9 (right panels) we show the spectra around the $\mathrm{Mg} \mathrm{I} b$ and $\mathrm{Na} I \mathrm{D}$ absorption features for two sources with enhanced $\mathrm{EW}(\mathrm{Na}$ I D). While the magnesium profile is well fitted by pPXF procedure (orange curves), the $\mathrm{Na} \mathrm{I} \mathrm{D} \mathrm{absorption} \mathrm{is} \mathrm{strongly} \mathrm{un-}$ derestimated. In panels [II] and [IV] we show our fitting decomposition of the He I - Na I D system after removing the stellar continuum. Mkn 848 exhibits a broad absorption profile with a $V_{\text {max }} \approx 1250 \mathrm{~km} \mathrm{~s}^{-1}$, which is even higher than the maximum velocity associated with the ionized outflowing gas $\left(\approx 900 \mathrm{~km} \mathrm{~s}^{-1}\right)$. For this source, the presence of neutral outflow on kpc scale with a substantial mass rate $\left(\dot{M}_{\text {out }}^{\mathrm{NaID}} \approx 100 M_{\odot} / \mathrm{yr}\right)$ has been proved by spatially resolved spectroscopic analysis (Rupke \& Veilleux 2013b).

The almost complete absence of neutral outflows in our sample is consistent with the results found by Villar-Martín et al. (2014), who reported the lack of atomic outflows in a sample of 21 SDSS type 2 QSOs with detected ionized outflows. Instead, the fraction of outflows in $\mathrm{Na}$ I D increases up to $45 \%$ in Seyfert ULIRGs (Rupke et al. 2005a) and in sources exhibiting both SF and AGN activity (Rupke et al. 2005b; Sarzi et al. 2016). In the context of an evolutionary scenario, these results suggest that the atomic outflows may be associated with earlier stages of the feedback phase, when the SF has not yet been completely inhibited and the AGN is still buried in dust enshrouded environments. This argument may explain the low incidence of atomic outflows and, on the whole, the low contribution of ISM absorption in our sample (see Fig. 9, left), which is characterized by a large number of unobscured type 1 AGNs and, in general, by sources with high level of ionization $(\log [\mathrm{O}$ III $] / \mathrm{H} \beta \sim 1$; Fig. 5) as per their selection. The X-ray/SDSS target with atomic 

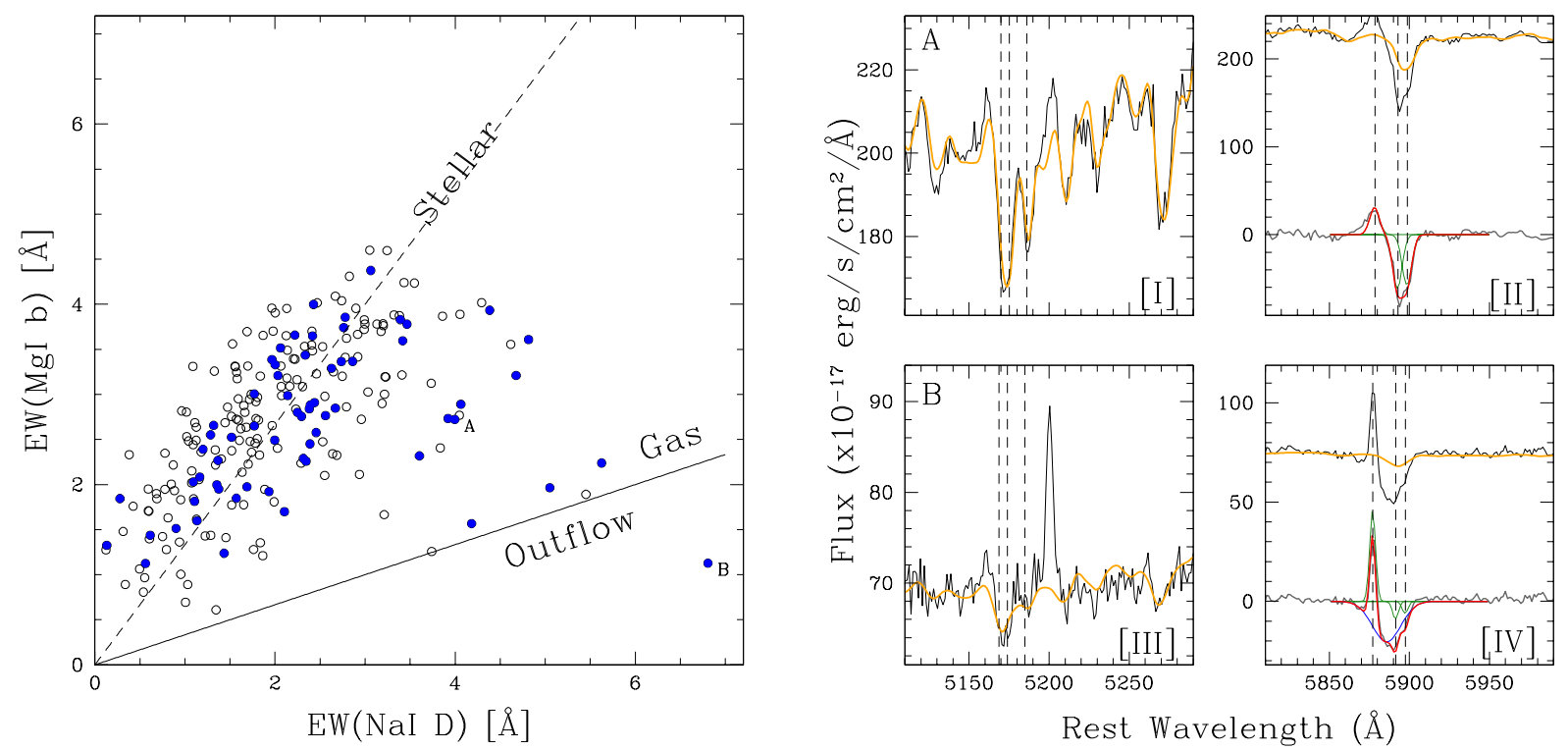

Fig. 9. Left: EW of the Na I D vs. Mg I b absorption lines. Open black and solid blue points represents sources with and without ionized outflows respectively (i.e. $V_{\max } \lessgtr 650 \mathrm{~km} \mathrm{~s}^{-1}$ ). The dashed line indicates the ratio at which the sodium is expected on the basis of observed magnesium EW (Heckman et al. 2000); the solid line indicates the starburst-driven outflow region proposed by Rupke et al. (2005a). The letters A and B indicate the sources for which the spectra are shown on the right. Right: SDSS spectra of J113240.24+525701.3 and MKN 848 (indicated with the letters A and $\mathrm{B}$ in the diagnostic diagram) around the $\mathrm{Mg} \mathrm{I} \mathrm{b} \mathrm{(left} \mathrm{panels)} \mathrm{and} \mathrm{Na} \mathrm{I} \mathrm{D} \mathrm{(right} \mathrm{panels)} \mathrm{absorption} \mathrm{features.} \mathrm{Orange} \mathrm{curves} \mathrm{show} \mathrm{the} \mathrm{best-fit}$ pPXF results. Vertical lines represent the systemic of $\mathrm{Mg} \mathrm{I} b$ triplet ([I] and [III] panels), and He I and Na I D doublet ([II] and [IV] panels). Panels [II] and [IV] show our fit results. Green, blue, and red curves represent the NC, OC, and the total best-fit model, respectively.

(and ionized) outflow signatures, Mkn 848, is a luminous infrared galaxy at $z=0.04$ with high SFR $\left(120 M_{\odot} /\right.$ yr $)$ associated with an ongoing merger (Rupke \& Veilleux 2013b), sharing therefore the same characteristics of other sources with unveiled neutral outflows. For example, Mkn 848 shares the same properties of other local ULIRG-QSO systems, such as Mrk 231, in which both cold (neutral and molecular) and warm outflows have been revealed in its host galaxy (see Feruglio et al. 2015 and references therein). Furthermore, it is similar to other high- $z$ sources analysed by our group (Perna et al. 2015a; Cresci et al. 2015a): our analysis of the two quasars at $z \sim 1.5$, XID 2028 and XID 5321 actually showed concomitant intense star formation activity $\left(S F R \approx 250 M_{\odot} / y r\right)$ and outflowing processes involving both atomic and ionized gas.

\section{X-ray loudness}

In this section we derive the X-ray bolometric corrections $\left(k_{\mathrm{bol}}\right)$ to test the X-ray loudness role in the outflow phenomena. We derive the bolometric corrections by computing the ratio between the intrinsic X-ray luminosity and $L_{\text {bol }}$, as derived in Sect. 4. We compare our results with the relation found by Lusso et al. (2012), which these authors derived by studying the X-ray selected AGN population in the COSMOS field.

Figure 10 (left) shows the derived $k_{\text {bol }}$ against the AGN bolometric luminosities for our X-ray/SDSS sample (squared symbols). In the figure, large circles denote BL AGNs. This subsample is likely associated with more reliable $L_{\text {bol }}$ estimates, which are the values relative to the low-luminosity population corrected for host contamination (see Sect. 4). Therefore, we focus the analysis on the BL subsample, which, however, includes $80 \%$ of the entire sample. For these sources, we differentiate between targets with kinematic indication of outflows in the [O III] line (with $V_{\max }>650 \mathrm{~km} \mathrm{~s}^{-1}$ ), colour-coded for increasing velocities, and those with narrow [O III] profiles (empty circles).
From a visual inspection, it appears that the X-ray/SDSS sources are broadly distributed over the entire plane, regardless the presence/absence of signatures of outflows in their ionized gas component. To simplify the visualization, we also show in Fig. 10 (right) the source distribution normalizing (in log space) the $k_{\mathrm{bol}}$ with the value expected from the Lusso et al. (2012) relation $\left(k_{\text {bol-L12 }}\right.$; black curve in the left panel), considering only the luminosity range in which the relation has been calibrated (i.e. $\log \left(L_{\mathrm{bol}} / L_{\odot}\right)$ in the range $\left.10.8-13.5\right)$. The histogram shows that the entire sample of BL AGNs (grey curve) actually follows the expected relation for X-ray selected AGNs, displaying a Gaussian distribution. Most importantly, the distributions of sources with/without outflows are similar, i.e. we do not report any excess in the number of sources with outflows at negative $\log \left(k_{\mathrm{bol}} / k_{\mathrm{bol}-\mathrm{L} 12}\right)$. This result therefore seems to disprove the idea of an X-ray loudness associated with the feedback phase.

The $k_{\text {bol }}$ correction derived for the sources presented in our previous works (Brusa et al. 2015, 2016; Perna et al. 2015a), and for which we were able to derive well-constrained bolometric luminosities (from SED fitting technique) and intrinsic X-ray emission (fitting XMM spectra), are very solid. Although the $\mathrm{X}$-ray/SDSS $k_{\text {bol }}$ corrections are probably less reliable, the almost symmetric distribution with respect to the expected relation found by Lusso et al. (2012) suggests that we are actually able to derive good estimates for the X-ray bolometric corrections ${ }^{5}$. It is possible therefore that lower $k_{\mathrm{bol}}$ corrections are found in the

5 Figure 10 shows that X-ray/SDSS sources are characterized by more scattered distribution if compared with those of Lusso et al. (2012). This is reasonably due to our approach in deriving bolometric luminosities, which requires an empirical relation based on monochromatic luminosity or, for fainter objects, two empirical relations (see Sect. 4). At lower luminosities $\left(\log \left(L_{\mathrm{bol}} / L_{\odot}\right)<11.5\right)$ in the figure), $L_{\mathrm{bol}}$ and $k_{\mathrm{bol}}$ errors are of the order of a factor of $\sim 2$; at higher luminosities, they are of the order of $10-30 \%$. 

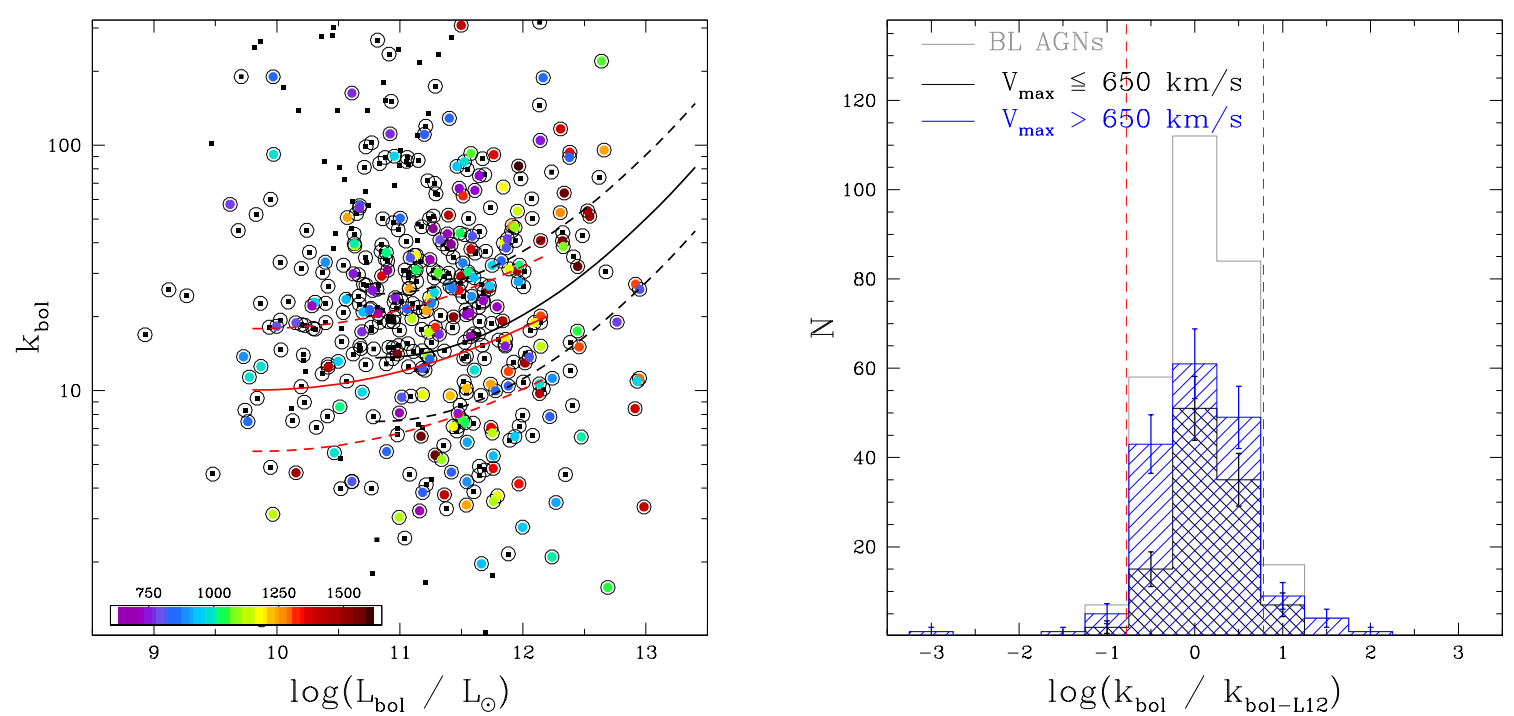

Fig. 10. Left panel: plot of $k_{\mathrm{bol}}$ vs. $L_{\mathrm{bol}}$. Black square symbols represent type 2 AGNs, while colour-coded circles indicate the type 1 and type 1.9 sources for increasing outflow velocity. The black and red curves represent the best-fitting relations obtained by Lusso et al. (2012) for type 1 and type 2 AGNs in the COSMOS field, respectively. Right: distribution of the normalized (in log space) $k_{\mathrm{bol}}$ values with respect to those expected from the relation for X-ray selected AGNs (black curve in the left panel) for the BL X-ray/SDSS sample (grey histogram). Blue and black histograms represent the distributions for sources with and without outflow signatures. Error bars indicate Poissonian errors. For comparison, $3 \times \sigma$ dispersion values derived by Lusso et al. (2012) for XMM-COSMOS sources are also shown with red dashed vertical lines.

high- $z$ sample because the sources of the two samples trace two different evolutionary phases with distinct properties.

The different incidence of atomic outflows between the X-ray/SDSS sample dominated by unobscured AGNs and the high- $z$ X-ray (and optical) obscured QSOs sample points in the same direction. In such a context, the X-ray/optical obscured AGNs studied in the previous works are associated with the initial stages of the feedback when atomic gas is still present in the ISM and outflow processes involve all the gas components (e.g. XID 2028 and XID 5321). The BL AGNs, instead, trace later stages, in which the line of sight has been cleaned and the cold components have been heated or exhausted. We note, however, that our suggestions are still based on small samples of obscured QSOs and that further investigation is needed to understand if the observed physical conditions at larger scales involving neutral ISM gas are somehow related with the accretion processes responsible for the X-ray emission.

\section{Summary}

We analysed SDSS optical and X-ray spectra of a large sample of 563 AGN at $z<0.8$ comprising type 1 (362), type 1.9 (77), and type 2 (124) sources. We combined ionized emission line and neutral absorption feature information modelled through multicomponent simultaneous fitting, non-parametric measurement, and pPXF analysis to derive physical (e.g. ionized levels) and kinematic conditions of both warm and cold gas components of the ISM. The main results are reported below.

Analysing the [O III] line profiles, we derived the outflow incidence in the warm phase of the NLR. We found that $\approx 40 \%$ of AGNs exhibit signatures of outflows. This fraction is strongly dependent on the AGN power; our analysis suggests a clear positive correlation between the outflow velocity and the AGN power traced by the [O III] luminosity. Such a trend has already been reported in the literature (e.g. Bae \& Woo 2014) and is also related to similar correlations between radio luminosity and [O III] width (e.g. Mullaney et al. 2013; Zakamska et al. 2016; Woo et al. 2016). However, the observed correlation between radio luminosity and stellar mass (and stellar velocity dispersion) may easily explain the trend with the [O III] widths, resulting in a challenging interpretation (see e.g. the discussion in Woo et al. 2016; Zakamska et al. 2016). We show instead, for the first time in a large sample ( 550 AGNs), that a well-defined positive trend of outflow velocity is observed with the unobscured $2-10 \mathrm{keV} \mathrm{X}$-ray luminosity, which is a tracer unambiguously associated with AGN activity, and it holds over 5 order of magnitudes.

Broad absorption line (BAL) quasars are generally associated with AGN-driven winds expelling UV/optical absorbing material at velocities such high as $\sim 40000 \mathrm{~km} \mathrm{~s}^{-1}$ (e.g. Dunn et al. 2010; Kaastra et al. 2014). This class of objects has been recently associated with intrinsically weak X-ray sources (e.g. Luo et al. 2014). The simplest explanation for the discrepancy between these results and the positive correlation we found between [O III] outflow velocity and X-ray luminosity is that UV/optical absorbing ejected material and ionized outflows may be related to different physical processes happening at different spatial scales (see e.g. Fiore et al. 2017).

The sodium absorption system at $\lambda \lambda 5890,5896$ was analysed to infer the presence of atomic outflowing gas in the ISM. We found signatures of atomic outflow in only one target and derived an incidence for the atomic outflows much lower $(<1 \%)$ than that obtained for the ionized counterpart.

We derived the X-ray bolometric correction and proved that the X-ray/SDSS sample characterized by the presence of ionized outflows does not show any deviation from the typical behaviour of the population of X-ray selected AGNs. This result may rule out the proposed role of X-ray emission in the feedback phase (Sect. 7; Brusa et al. 2016). However, our selection criteria allowed the collection of a sample that is mostly dominated by BL AGNs which, in the framework of the evolutionary scenario, may be associated with a different stage of the blow-out phase (e.g. Hopkins et al. 2008). We therefore suggested a scenario that explains the variety of evidence of neutral/ionized outflows and nuclear properties (i.e. X-ray activity), invoking two different evolutionary stages within the blow-out phase: first, an 
M. Perna et al.: An X-ray/SDSS sample. I.

initial X-ray/optical obscured stage, in which the atomic gas is still present in the ISM and the outflow processes involve all the gas components and, second, a later stage associated with unobscured AGNs, in which the line of sight has been cleaned and the cold components have been heated or exhausted.

Acknowledgements. M.P., G.L. and M.B. acknowledge support from the FP7 Career Integration Grant "eEASy" ("SMBH evolution through cosmic time: from current surveys to eROSITA-Euclid AGN Synergies", CIG 321913). Support for this publication was provided by the Italian National Institute for Astrophysics (INAF) through PRIN-INAF-2014 ("Windy Black Holes combing galaxy evolution"). We thank the anonymous referee for his/her constructive comments on the paper. Funding for the Sloan Digital Sky Survey (SDSS) has been provided by the Alfred P. Sloan Foundation, the Participating Institutions, the National Aeronautics and Space Administration, the National Science Foundation, the US Department of Energy, the Japanese Monbukagakusho, and the Max Planck Society. The SDSS Web site is http://www.sdss.org/. The SDSS is managed by the Astrophysical Research Consortium (ARC) for the Participating Institutions. The Participating Institutions are The University of Chicago, Fermilab, the Institute for Advanced Study, the Japan Participation Group, The Johns Hopkins University, Los Alamos National Laboratory, the Max-Planck-Institute for Astronomy (MPIA), the Max-Planck-Institute for Astrophysics (MPA), New Mexico State University, University of Pittsburgh, Princeton University, the United States Naval Observatory, and the University of Washington.

\section{References}

Abazajian, K. N., Adelman-McCarthy, J. K., Agüeros, M. A., et al. 2009, ApJS, 182,543

Arribas, S., Colina, L., Bellocchi, E., et al. 2014, A\&A, 568, A14

Bae, H., \& Woo, J. 2014, ApJ, 795, 30

Baldwin, J. A., Phillips, M. M., \& Terlevich, R. 1981, PASP, 93, 5

Balmaverde, B., Marconi, A., Brusa, M., et al. 2016, A\&A, 585, A148

Bentz, M. C., Denney, K. D., Grier, C. J., et al. 2013, ApJ, 767, 149

Bongiorno, A., Maiolino, R., Brusa, M., et al. 2014, MNRAS, 443, 2077

Bouché, N., Finley, H., Shroetter, I., et al. 2016, ApJ, 820, 121

Brusa, M., Bongiorno, A., Cresci, G., et al. 2015, MNRAS, 446, 2394

Brusa, M., Perna, M., Cresci, G., et al. 2016, A\&A, 588, A58

Cano-Díaz M. R., Marconi, A., Netzer, H., Shemmer, O., \& Cresci, G. 2012, A\&A, 537, L8

Cappellari, M. 2017, MNRAS, 466, 798

Cappellari, M., \& Emsellem, E. 2004, PASP, 116, 138

Carniani, S., Marconi, A., Maiolino, R., et al. 2015, A\&A, 580, A102

Cicone, C., Maiolino, R., Sturm, E., et al. 2014, A\&A, 562, A21

Cimatti, A., Brusa, M., Talia, M., et al. 2013, ApJ, 779, 13

Collet, C., Nesvadba, N. P. H., De Breuck, C., et al. 2016, A\&A, 586, A152

Concas, A., Popesso, P., Brusa, M., et al. 2017, A\&A, accepted DOI: $10.1051 / 0004-6361 / 201629519$

Cresci, G., Mainieri, V., Brusa, M., et al. 2015a, ApJ, 799, 81

Cresci G., Marconi, A., Zibetti, S., et al. 2015b, A\&A, 582, A63

De Robertis, M. M., \& Osterbrock, D. E. 1986, ApJ, 301, 727

Dunn, J., Crenshaw, D. M., Kraemer, S. B., et al. 2010, ApJ, 713, 900

Fabian, A. C. 2012, ARA\&A, 50, 455

Feruglio, C., Maiolino, R., Piconcelli, E., et al. 2010, A\&A, 518, A155

Feruglio, C., Fiore, F., Carniani, S., et al. 2015, A\&A, 583, A99

Fiore, F., Feruglio, C., Shankar, F., et al. 2017, A\&A, 601, A143

Fischer, T. C., Crenshaw, D. M., Kraemer, S. B., \& Schmitt, H. R. 2013, ApJS, 209, 1

Georgakakis, A., \& Nandra, K. 2011, MNRAS, 414, 992

Glikman, E., Urrutia, T., Lacy, M. et al. 2012, ApJ, 757, 51

Greene, J. E., \& Ho, L. C. 2005, ApJ, 630, 122

Harrison, C. M., Alexander, D. M., Swinbank, A. M., et al. 2012, MNRAS, 426, 1073
Harrison, C. M., Alexander, D. M., Mullaney, J. R., et al. 2014, MNRAS, 441, 3306

Harrison, C. M., Alexander, D. M., Mullaney, J. R., et al. 2016, MNRAS, 456, 1195

Heckman, T. M., Lehnert, M. D., Strickland, D. K., \& Armus L. 2000, ApJS, 129,493

Heckman, T. M., Kauffmann G., Brinchmann J., et al. 2004, ApJ, 613, 109

Hopkins, P. F., Hernquist, L., Cox, T. J., \& Kereš, D. 2008, ApJS, 175, 356

James, F., \& Roos, M. 1975, Comput. Phys. Commun., 10, 343

Jin, C., Ward, M., Done, C., et al. 2012, MNRAS, 420, 1825

Kaastra, J., Kriss, J. A., Cappi, M., et al. 2014, Science, 345, 64

Kakkad, D., Mainieri, V, Padovani, P., et al. 2016, A\&A, 592, A148

Kaspi, S., Maoz, D., Netzer, H., et al. 2005, ApJ, 629, 61

Kewley, L. J., Maier, C., Yabe, K., et al. 2013, ApJ, 774, L10

King, A., \& Pounds, K. 2015, ARA\&A, 53, 115

Komossa, S., Xu, D., Zhou, H., et al. 2008, ApJ, 680, 926

Kovačević, J., Popović, L. Č., \& Dimitrijević, M. S. 2010, ApJS, 189, 15

Lanzuisi, G., Civano, F., Elvis, M., et al. 2013, MNRAS, 431, 978

Lanzuisi, G., Perna, M., Delvecchio, I., et al. 2015, A\&A, 578, A120

Liu, G., Zakamska, N. L., Greene, J. E., et al. 2013, MNRAS, 436, 2576

Luo, B., Brandt, W. N., Alexander, D. M., et al. 2014, ApJ, 794, 70

Lusso, E., Comastri, A., Simmons, B. D., et al. 2012, MNRAS, 425, 623

McElroy, R., Croom, S. M., Pracy, M., et al. 2015, MNRAS, 446, 2186

Menci, N., Fiore, F., Puccetti, S., \& Cavaliere, A. 2008, ApJ, 686, 219

Merloni, A., Bongiorno, A., Brusa, M. et al. 2014, MNRAS, 437, 3550

Mullaney, J. R., Alexander, D. M., Fine, S., et al. 2013, MNRAS, 433, 622

Mullaney, J. R., Alexander, D. M., Aird, J., et al. 2015, MNRAS, 453, 83

Müller-Sánchez, F., Prieto, M. A., Hicks, E. K. S., et al. 2011, ApJ, 739, 69

Netzer, H., \& Trakhtenbrot, B. 2014, MNRAS, 438, 672

Osterbrock, D. E., \& Ferland, G. J. 2006, Astrophysics of Gaseous Nebulae and Active Galactic Nuclei (University Science Books)

Panessa, F., Bassani, L., Cappi, M., et al. 2006, A\&A, 455, 173

Perna, M., Brusa, M., Cresci, G., et al. 2015a, A\&A, 574, A82

Perna, M., Brusa, M., Salvato, M., et al. 2015b, A\&A, 583, A72

Planck Collaboration XVI. 2014, A\&A, 571, A16

Press, W. H., Teukolsky, S. A., Vetterling, W. T., \& Flannery, B. P. 1992, Numerical recipes in Fortran, 2nd edn. (New York: Cambridge University Press)

Reyes, R., Zakamska, N., Strauss, M. A., et al. 2008, AJ, 136, 2373

Rupke, D. S. N., \& Veilleux, S. 2013a, ApJ, 775, 15

Rupke, D. S. N., \& Veilleux, S. 2013b, ApJ, 768, 75

Rupke, D. S., Veilleux, S., \& Sanders, D. B. 2005a, ApJS, 160, 87

Rupke, D. S., Veilleux, S., \& Sanders, D. B. 2005b, ApJS, 160, 115

Sarzi, M., Kaviraj, S., Nedelchev, B., et al. 2016, MNRAS, 456, 25

Saturni, F. G., Trevese, D., Vagnetti, F., et al. 2016, A\&A, 587, A43

Schawinski, K., Koss, M., Berney, S., \& Sartori, L. F. 2015, MNRAS, 451, 2517

Shen, Y., \& Kelly, B. C. 2012, ApJ, 746, 169

Shen, Y., Richards, G. T., Strauss, M. A., et al. 2011, ApJS, 194, 45

Sturm, E., González-Alfonso, E., Veilleux, S., et al. 2011, ApJ, 733, L16

Talia, M., Cimatti, A, Brusa, M., et al. 2016, MNRAS, submitted [arXiv: 1611.05884]

Thomas, D., Steele, O., Maraston, C., et al. 2013, MNRAS, 431, 1383

Trichas, M., Green, P. J., Constantin, A., et al. 2013, ApJ, 778, 188

Veilleux, S., Meléndez, M., Sturm, E., et al. 2013, ApJ, 776, 27

Véron-Cetty, M. P., Véron, P., \& Gonçalves, A. C. 2001, A\&A, 372, 730

Vestergaard, M., \& Peterson, B. M. 2006, ApJ, 641, 689

Villar-Martín M., Emonts, B., Humphrey, A., et al. 2014, MNRAS, 440, 3202

Watson, M. G., Schröder, A. C., Fyfe, D., et al. 2009, A\&A, 493, 339

Wang, J., \& Xu, D. W. 2015, A\&A, 573, A15

Westmoquette, M. S., Clements, D. L., Bendo, G. J., \& Khan, S. A. 2012, MNRAS, 424, 416

Woo, J. H., Bae, H. J., Son, D., \& Karouzos, M. 2016, ApJ, 817, 108

Wu, J., Vanden Berk, D., Grupe, D., et al. 2012, ApJS, 201, 10

Wylezalek, D., \& Zakamska, N. L. 2016, MNRAS, 461, 3724

Yuan, S., Strauss, M. A., \& Zakamska, N. L. 2016, MNRAS, 462, 1603

Zakamska, N. L., \& Greene, J. E. 2014, MNRAS, 442, 784

Zakamska, N. L., Lampayan, K., Petric, A., et al. 2016, MNRAS, 455, 4191 


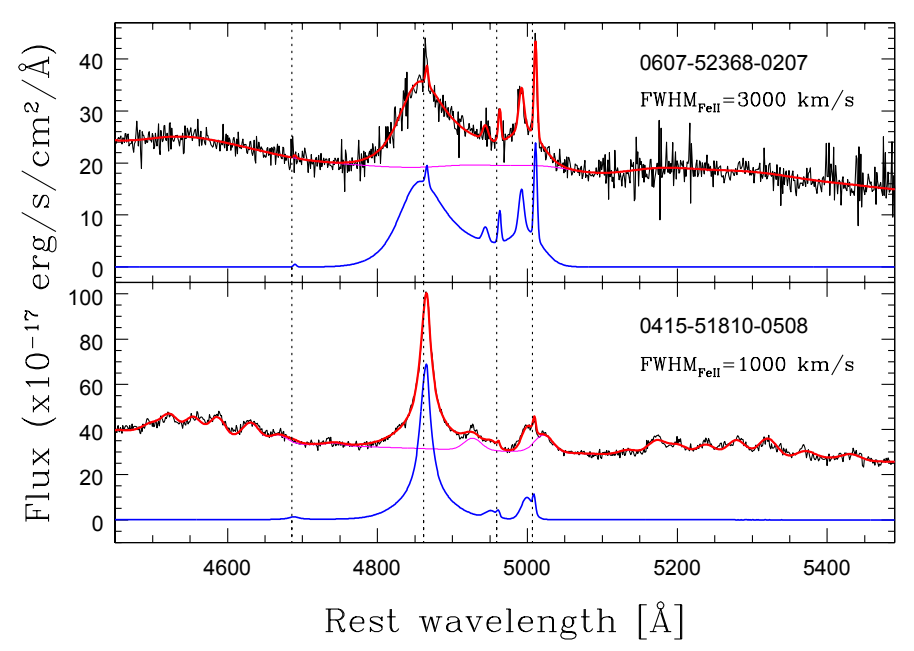

Fig. A.1. Multicomponent simultaneous best-fit results (red curves) for two X-ray/SDSS sources showing iron emission and double peaked $[\mathrm{O} I I I]$ profiles. Black profiles represent rest-frame SDSS spectra. Blue and magenta curves indicate the best-fit results for the [O III]- $\mathrm{H} \beta$ emission and for the Fe II lines, respectively. For visual inspection, the iron profile is added to the power-law continuum. The figure shows the deconvolution between Fe II and the reddest emission from [O III]. We labelled in the panels the MJD, the plate, and the fibre numbers, which uniquely identifies the SDSS spectra and FWHM required to fit the iron emission.

\section{Appendix A: Fe II emission line template}

We use theoretical model templates of Kovačević et al. (2010) to reproduce Fe II emission. The authors constructed a Fe II template consisting of tens of line components, identified as the strongest within 4000-5500 A range. Many of these line components are sorted into five line groups according to the lower term of their atomic transition (P, F, S, G, and H; see their Fig. 1). Relative intensities of each Fe II Gaussian line within a single group have been calculated by studying the transition probabilities. A sixth group, whose relative intensities have been obtained on the basis of their best fit of the well-studied I Zw 1 spectrum, was added to reproduce the overall Fe II profile. These templates assume seven free fitting parameters: the shift relative to the systemic redshift and the FWHM of the Fe II lines, which are assumed to be the same for all the template lines, and the intensities of the Fe II lines within the line groups. Iron emission strongly covers the entire wavelength range of $\mathrm{H} \beta$ and [OIII]. As a result, a correct modelling is needed to fully characterize the Balmer profile, and in particular its FWHM, from which crucial information can be derived, and the double ionized oxygen emission. One of the $S$ group lines (see Kovačević et al. 2010, Fig. 1) may be responsible for a red excess in the [O III] line, leading to a wrong determination of the kinematic conditions in the ionized gas. The fact that the amplitude of this line is linked to the intensity of the entire $S$ group (and, in particular, to the nearest line in the red Fe II bump) gives an important contribution to the deconvolution between Fe II and reddest emission from [O III]. As an example, we show in Fig. A.1 the fitting results we obtain for a couple of SDSS sources, in which prominent red wings in the $[\mathrm{O}$ III] line are detected.

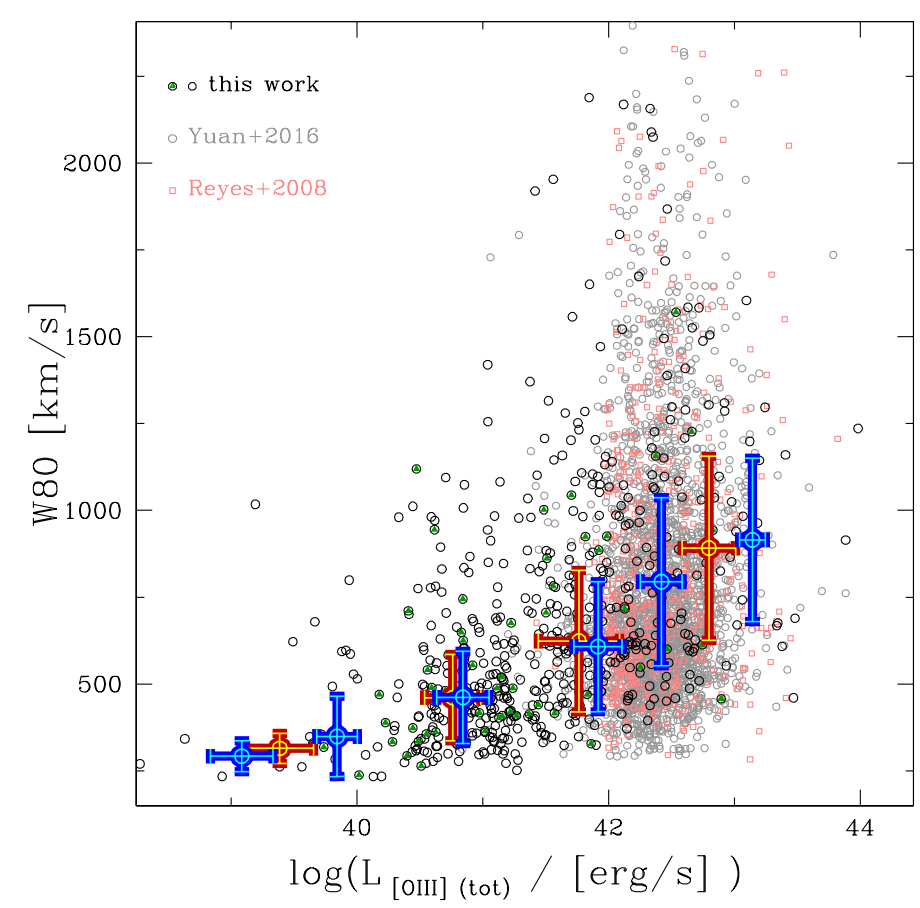

Fig. B.1. Non-parametric velocity $W 80$ estimator as a function of [O III] luminosity. Black and green/black symbols refer to the same $\mathrm{X}$-ray/SDSS sources shown in Fig 8; grey circles and pink squares shows type 2 AGNs from Yuan et al. (2016) and Reyes et al. (2008). Large symbols represent median velocities in bins of luminosity for the X-ray/SDSS sample (red/yellow) and for all sources in the figure (blue/cyan); bars indicate semi-interquartile ranges. Yuan et al. and Reyes et al. luminosities have been recomputed consistently with the cosmological parameters shown in the Introduction.

\section{Appendix B: [O III] velocity - luminosity trend. Supporting evidence from literature}

In Fig. B.1 we compare our results with those obtained studying other large samples of SDSS type 2 AGNs in the same redshift range and for which non-parametric $W 80$ velocity estimates are available 6 . Yuan et al. $(2016, \sim 2900)$ and Reyes et al. $(2008, \sim 550)$ sources are shown with grey and pink symbols, respectively. Their luminosity ranges $\left(\sim 10^{42}-10^{43} \mathrm{erg} / \mathrm{s}\right)$ are much shorter than those covered by our X-ray/SDSS sources and do not permit a real comparison of velocity - luminosity trends; however, when we derive the median values in luminosity bins for our sources (red/yellow large symbols) and for the total sample obtained combining our sources with theirs (blue/cyan large symbols), we do not observe any deviation at higher luminosities $\left(\gtrsim 10^{42} \mathrm{erg} / \mathrm{s}\right)$. The large number $(\sim 4000)$ of sources, together with the use of median instead of average values in luminosity bins (less sensitive to too much deviated velocity estimates), supports the results we have shown in Fig. 8.

\section{Appendix C: Signal-to-noise ratio and NC/OC flux ratio investigation}

In order to prove that the correlations shown in Sect. 5 are not due to detection biases, we investigated whether the absence of fast outflows at low luminosities and of slow outflows at highest luminosities are related to particular OC/NC flux ratios and/or $\mathrm{S} / \mathrm{N}$ of the OC Gaussians.

6 http://zakamska.johnshopkins. edu/data.htm 

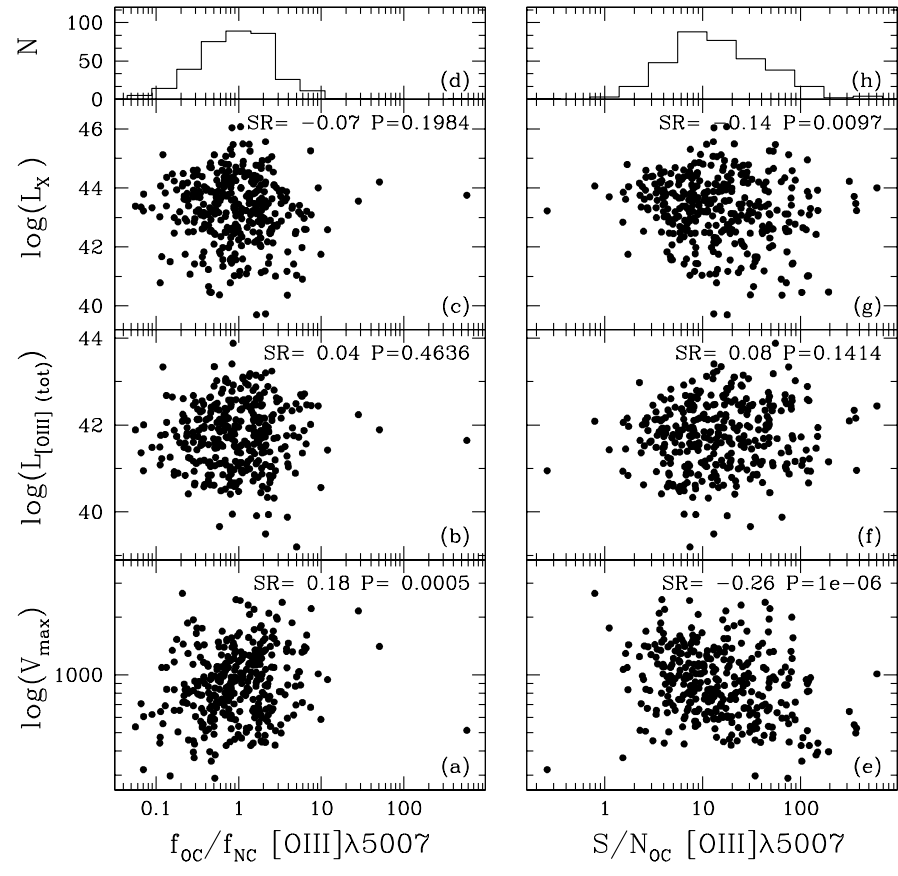

Fig. C.1. OC/NC flux ratios and $[\mathrm{O}$ III $] \lambda 5007$ OC signal to noise $\left(\mathrm{S} / \mathrm{N}_{\mathrm{OC}}\right)$ as a function of $V_{\max },[\mathrm{O} \mathrm{III}]$ and $\mathrm{X}$-ray luminosities for the subsample modelled with NC+OC Gaussians. Spearman rank coefficients and null hypothesis probabilities are also labelled in each panel. The panels in the first row indicate the distributions of $\mathrm{OC} / \mathrm{NC}$ ratios and $\mathrm{S} / \mathrm{N}_{\mathrm{OC}}$.

Figure C.1, panel a, shows the distribution of $f_{\mathrm{OC}} / f_{\mathrm{NC}}$ versus $V_{\max }$ (in log-space) for the sub-sample modelled with $\mathrm{NC}+\mathrm{OC}$ Gaussians. A poor positive correlation $(S R=0.18$ with null hypothesis $\sim 10^{-5}$ ) is observed. This correlation is expected as nonparametric velocity estimators are computed from total [O III] flux percentiles (Sect. 3) and, when broad prominent wings are present, both relative $\mathrm{OC} / \mathrm{NC}$ flux contributions and $V_{\max }$ are expected to increase. The correlation is inconspicuous because of the dependence of maximum velocity on the widths of Gaussian components, which are not taken into account here.
Figure C.1, panel e, shows the distribution of OC signal-tonoise ratio versus $V_{\max }$ (log-space). Also in this case, we observe a poor (negative) correlation $\left(S R=-0.26, P \approx 10^{-6}\right)$. The correlation suggests negligible effects of bias selections: naively, highest velocities could be uniquely associated with objects with prominent (i.e. well detected), extended wings. If this is the case, a positive correlation should be observed in our $S N_{\mathrm{OC}}-V_{\max }$ distribution. On the other hand, it is possible that the highest velocities could be incorrectly derived when [O III] wings are characterized by low $\mathrm{S} / \mathrm{N}$. We mitigated as best as possible this effect with simultaneous fit modelling (see Sect. 3); the poor correlation prove the capabilities of this technique.

Panels $b, c, f$, and $g$ show the distributions of OC/NC flux ratios and $\mathrm{S} / \mathrm{N}_{\mathrm{OC}}$ with respect to $[\mathrm{O} \mathrm{III}]$ and $\mathrm{X}$-ray luminosities. In these cases, we do not observe any relevant correlation $(S R \sim-0.1 \div 0.1, P \sim 1 \div 50 \%)$. We can therefore reasonably exclude any contribution of low $\mathrm{S} / \mathrm{N}$ or peculiar $\mathrm{OC} / \mathrm{NC}$ to the correlations shown in Sect. 5 between outflow velocity and AGN luminosity.

Finally, panels $\mathrm{d}$ and $\mathrm{h}$ show the distributions of $\mathrm{NC} / \mathrm{OC}$ and $\mathrm{S} / \mathrm{N}_{\mathrm{OC}}$. We note that the OC contribution to the total oxygen flux is, on average, of the same order of magnitude of NC flux. The $\mathrm{S} / \mathrm{N}_{\mathrm{OC}}$ distribution shows that the majority of our sample modelled with $\mathrm{NC}+\mathrm{OC}$ Gaussians also presents significant OC emission $(S / N>3)$. Only $\sim 20$ sources are below this threshold. We do not exclude any of these as the presence of OC Gaussians has also been proved in other emission lines in the $\mathrm{H} \beta$ and $\mathrm{H} \alpha$ regions. Also, only $\sim 15$ targets enter in the determination of the fraction of AGNs with outflows ( $V_{\max }>665 \mathrm{~km} \mathrm{~s}^{-1}$; Sect. 5). Therefore, their contribution does not determine significant variation in our results. 